\title{
Uniform dimension results for a family of Markov processes
}

\author{
XIAOBIN SUN ${ }^{1}$, YIMIN XIAO ${ }^{2}$, LIHU XU ${ }^{3,4,6}$ and JIANLIANG ZHAI \\ ${ }^{1}$ School of Mathematics and Statistics, Jiangsu Normal University, Xuzhou 221116, China. \\ E-mail:xbsun@jsnu.edu.cn \\ ${ }^{2}$ Department of Statistics and Probability, Michigan State University, East Lansing, MI 48824, USA. \\ E-mail:xiao@stt.msu.edu \\ ${ }^{3}$ Department of Mathematics, Faculty of Science and Technology, University of Macau, E11 Avenida da \\ Universidade,Taipa, Macau, China.E-mail: lihuxu@umac.mo \\ ${ }^{4}$ UM Zhuhai Research Institute, Zhuhai, China \\ ${ }^{5}$ School of Mathematical Science, University of Science and Technology of China, Hefei, 230026, China. \\ E-mail:zhaijl@ustc.edu.cn
}

In this paper, we prove uniform Hausdorff and packing dimension results for the images of a large family of Markov processes. The main tools are the two covering principles in Xiao (In Fractal Geometry and Applications: A Jubilee of Benoît Mandelbrot, Part 2 (2004) 261-338 Amer. Math. Soc.). As applications, uniform Hausdorff and packing dimension results for certain classes of Lévy processes, stable jump diffusions and non-symmetric stable-type processes are obtained.

Keywords: cover principles; Markov processes; uniform Hausdorff dimension

\section{Preliminaries}

Fractal properties of Brownian motion and more general Lévy processes have been studied extensively. We refer to the recent books of Mörters and Peres [42], Schilling and Partzsch [50], Böttcher, Schilling and Wang [8], the survey papers Taylor [52], Xiao [56], and more recent articles Khoshnevisan and Xiao [26,29], Knopova, Schilling and Wang [34] for further information.

Let $X=\left\{X(t), t \in \mathbb{R}_{+}\right\}$be a stable Lévy process in $\mathbb{R}^{d}$ of index $\alpha(0<\alpha \leq 2)$. For any Borel set $E \subseteq \mathbb{R}_{+}=[0, \infty)$, Blumenthal and Getoor [7] obtained the Hausdorff dimension of the image set $X(E)$, namely,

$$
\operatorname{dim}_{\mathrm{H}} X(E)=\min \left\{d, \alpha \operatorname{dim}_{\mathrm{H}} E\right\} \quad \text { a.s., }
$$

where $\operatorname{dim}_{H}$ denotes Hausdorff dimension; see Falconer [13], or Mörters and Peres [42], Taylor [52], Xiao [56] for the definitions and properties of Hausdorff measure and Hausdorff dimension.

Result (1.1) has been extended and strengthened in various directions by many authors; see Taylor [52], Xiao [56] and the references therein for a historical account and information on development on (mostly) Lévy processes. In particular, Hawkes and Pruitt [18], Theorem 4.1, established a uniform version of (1.1): If $X$ is a strictly stable Lévy process of index $\alpha$ in $\mathbb{R}^{d}$ and

${ }^{6}$ Corresponding author.

1350-7265 @ 2018 ISI/BS 
$d \geq \alpha$, then there exists a single null probability event outside of which (1.1) holds simultaneously for all Borel sets $E \subseteq \mathbb{R}_{+}$. The first such uniform dimension result was due to Kaufman [22] for planar Brownian motion (i.e., $\alpha=2$ and $d=2$ ). A short and easily accessible redaction of Kaufman's original argument can be found in Schilling and Partzsch [50]. Such uniform dimension results are useful in many situations because it allows $E$ to be a random set (cf. Benjamini, Chen and Rohde [4], Mörters and Peres [42] for some applications). Perkins and Taylor [44] further proved uniform Hausdorff and packing measure results for strictly stable Lévy processes. As a consequence, they proved a packing dimension analogue of Hawkes and Pruitt [18], Theorem 4.1: If $X$ is a strictly stable Lévy process of index $\alpha$ in $\mathbb{R}^{d}$ and $d \geq \alpha$, then with probability 1 ,

$$
\operatorname{dim}_{P} X(E)=\alpha \operatorname{dim}_{P} E \quad \text { for all Borel sets } E \subseteq \mathbb{R}_{+},
$$

where $\operatorname{dim}_{P}$ denotes packing dimension (cf. e.g., Falconer [13], Mörters and Peres [42], Taylor [52], Xiao [56]). Note that, a strictly $\alpha$-stable Lévy process is self-similar with index $H=1 / \alpha$, and is an important representative among self-similar processes and random fractals.

In recent years, there has been increasing interest in constructing and studying more general Markov processes related to Lévy processes. A large class of Markov processes are generated by pseudo-differential operators (Böttcher, Schilling and Wang [8], Jacob [19], Knopova and Kulik [32] Jacob and Schilling [20], Schilling and Schnurr [51]), their corresponding transition probabilities and heat kernel estimates have been studied in, for example, Kolokoltsov [35,36], Kühn [37,38], Negoro [43]. Also, we refer to Bass [3] for the martingale problem of pure jump Markov processes, Chen [10], Chen and Kumagai [11], Chen and Zhang [12] for stable-like processes related to Dirichlet forms and their heat kernel estimates. Many natural questions regarding sample path and fractal properties arise for such Markov processes. Xiao [56] gives a comprehensive survey on fractal properties of Lévy or more general Markov processes before 2004. A lot of progress has been made since then. See, for example, Khoshnevisan, Schilling and Xiao [24], Khoshnevisan and Xiao [25-29] for various results on Lévy processes, Chen [10], Chen and Kumagai [11], Yang [58] for Hausdorff dimension of the range or graph of stable-like processes, Knopova, Schilling and Wang [34], Knopova and Schilling [33] for results on Hausdorff dimensions of the image, level and collision sets of a class of Feller process generated by a pseudo-differential operator. However, many interesting problems described in Xiao [56] are still open.

The main purpose of this paper is to provide a general method for establishing uniform Hausdorff and packing dimension results for more general Markov processes. In particular, we extend the methods of Hawkes [16] and Hawkes and Pruitt [18] for stable Lévy processes (see also Pruitt [45]) to a large family of Markov processes including more general Lévy processes, the stable-like processes or stable jump diffusions in Chen and Kumagai [11], Chen and Zhang [12], Kolokoltsov [35], Negoro [43]. The key technical tools are the covering principles in Section 2, which improve Lemmas 8.1 and 8.2 in Xiao [56]. We apply them to show the main result of this paper, Theorem 3.3, in Section 3. In Section 4, we apply Theorem 3.3 to Lévy processes and stable-type processes. We mention that Benjamini, Chen and Rohde [4] studied the normally reflected Brownian motion (RBM) in a class of non-smooth domains and proved their uniform Hausdorff dimension result by using the uniform Hölder continuity of RBM, the aforementioned 
Kaufman's theorem for Brownian motion and a subordination argument. Moreover, they indicated in Remark 3.10 in Benjamini, Chen and Rohde [4] that similar result still holds for the stable-like processes in Chen and Kumagai [11] by using the covering principle in Xiao [56]. The scope of the present paper is a lot broader, and we expect that the main result of this paper will also be useful for studying fractal sets related to intersections and multiple points of Markov processes.

In the rest of this paper, we assume that $X=\left\{X(t), t \in \mathbb{R}_{+}, \mathbb{P}^{x}\right\}$ is a time-homogeneous Markov process with state space $\mathbb{R}^{d}$, defined on some probability space $(\Omega, \mathcal{F}, \mathbb{P})$, and satisfies the strong Markov property. We assume that $X$ is separable and its sample paths are almost surely right continuous and have left limit at every $t \in \mathbb{R}_{+}$(such a sample function will be called cadlag).

An unspecified positive and finite constant will be denoted by $C$, which may be different in each appearance. More specific constants are numbered by $K_{1}, K_{2}, \ldots$ and $C_{1}, C_{2}, \ldots$

\section{The covering principles}

In the review article Xiao [56], Lemmas 8.1 and 8.2, Xiao stated without proof two covering principles that extend respectively Lemma 3.1 in Hawkes and Pruitt [18] and Lemma 3 in Hawkes [16] for Lévy processes (see also Lemmas 1 and 2 in Pruitt [45]), and suggested that they are useful for proving uniform Hausdorff and packing dimension results for the images of a general Markov process. In this section, we weaken the conditions of Lemmas 8.1 and 8.2 in Xiao [56] and provide proofs.

The following lemma is useful for proving upper bounds for the Hausdorff and packing dimensions of the image of a Markov process. Its proof is a modification of that of Lemma 3.1 in Hawkes and Pruitt [18], and we provide it for the sake of completeness. Moreover, in Proposition 3.1 below, we will provide a convenient way to verify condition (2.1).

Lemma 2.1. Let $X=\left\{X(t), t \in \mathbb{R}_{+}, \mathbb{P}^{x}\right\}$ be a time homogenous strong Markov process in $\mathbb{R}^{d}$. Let $\left\{t_{n}, n \geq 1\right\}$ be a sequence of positive real numbers such that $\sum_{n=1}^{\infty} t_{n}^{p}<\infty$ for some $p>0$, and let $\mathcal{C}_{n}$ be a class of $N_{n}$ intervals in $\mathbb{R}_{+}$of length $t_{n}$ with $\log N_{n}=O(1)\left|\log t_{n}\right|$. If there is a sequence $\left\{\theta_{n}\right\}$ of positive numbers such that for all $x \in \mathbb{R}^{d}$,

$$
\mathbb{P}^{x}\left\{\sup _{0 \leq s \leq t_{n}}|X(s)-x| \geq \theta_{n}\right\} \leq K_{1} t_{n}^{\delta},
$$

where $K_{1}$ and $\delta$ are some positive constants, then there exists a positive integer $K_{2}$, depending on $p$ and $\delta$ only, such that, with $\mathbb{P}^{x}$-probability one, for $n$ large enough, $X(I)$ can be covered by $K_{2}$ balls of radius $\theta_{n}$ whenever $I \in \mathcal{C}_{n}$.

Proof. Let $I \in \mathcal{C}_{n}$ and write it as $I=\left[a, a+t_{n}\right]$. Let $\tau_{0}=a$ and, for all $j \geq 1$, define

$$
\tau_{j}=\inf \left\{s>\tau_{j-1}:\left|X_{s}-X_{\tau_{j-1}}\right|>\theta_{n}\right\},
$$

with the convention inf $\varnothing=\infty$. It is easy to see

$$
\left\{X(I, \omega) \text { cannot be covered by } k \text { balls of radius } \theta_{n}\right\} \subseteq\left\{\tau_{k}-\tau_{0} \leq t_{n}\right\} .
$$


Moreover, by the strong Markov property and (2.1),

$$
\begin{aligned}
\mathbb{P}^{x}\left\{\tau_{k}-\tau_{0} \leq t_{n}\right\} & \leq \mathbb{E}^{x}\left\{\mathbb{E}^{x}\left[1_{\left\{\tau_{k}-\tau_{k-1} \leq t_{n}\right\}} 1_{\left\{\tau_{k-1}-\tau_{0} \leq t_{n}\right\}} \mid \mathcal{F}_{\tau_{k-1}}\right]\right\} \\
& =\mathbb{E}^{x}\left\{\mathbb{E}^{x}\left[1_{\left\{\tau_{k}-\tau_{k-1} \leq t_{n}\right\}} \mid \mathcal{F}_{\tau_{k-1}}\right] 1_{\left\{\tau_{k-1}-\tau_{0} \leq t_{n}\right\}}\right\} \\
& \leq \sup _{y \in \mathbb{R}^{d}} \mathbb{P}^{y}\left\{\sup _{0 \leq s \leq t_{n}}|X(s)-y| \geq \theta_{n}\right\} \mathbb{E}^{x}\left\{1_{\left\{\tau_{k-1}-\tau_{0} \leq t_{n}\right\}}\right\} \\
& \leq K_{1} t_{n}^{\delta} \mathbb{P}^{x}\left\{\tau_{k-1}-\tau_{0} \leq t_{n}\right\}
\end{aligned}
$$

Using the above argument recursively, we obtain that for all $n \geq 1$,

$$
\mathbb{P}^{x}\left\{\tau_{k}-\tau_{0} \leq t_{n}\right\} \leq K_{1}^{k} t_{n}^{k \delta}
$$

Define events

$$
A_{n}^{k}:=\left\{\exists I \in \mathcal{C}_{n} \text { such that } X(I, \omega) \text { cannot be covered by } k \text { balls of radius } \theta_{n}\right\} .
$$

Since $\log N_{n}=O(1)\left|\log t_{n}\right|$, i.e., there exist positive constant $C$, such that $N_{n} \leq C t_{n}^{-C}$, as $k$ is large enough (say, $\delta k-C \geq p$ ), we obtain from (2.3) that

$$
\begin{aligned}
\sum_{n=1}^{\infty} \mathbb{P}^{x}\left(A_{n}^{k}\right) & \leq \sum_{n=1}^{\infty} N_{n} K_{1}^{k} t_{n}^{k \delta} \\
& \leq C K_{1}^{k} \sum_{n=1}^{\infty} t_{n}^{-C+\delta k} \leq C K_{1}^{k} \sum_{n=1}^{\infty} t_{n}^{p}<\infty
\end{aligned}
$$

Hence, the Borel-Cantelli lemma yields the desired result. The proof is complete.

For obtaining lower bounds for the Hausdorff and packing dimensions of the image of a Markov process, one can apply the following lemma. Observe that the condition (2.4) is significantly weaker than (8.7) in Lemma 8.2 in Xiao [56] (which is usually satisfied only if $X$ is transient) and is easier to verify (see (3.15) below).

Lemma 2.2. Let $X=\left\{X(t), t \in \mathbb{R}_{+}, \mathbb{P}^{x}\right\}$ be a time homogenous strong Markov process in $\mathbb{R}^{d}$. Let $\left\{r_{n}, n \geq 1\right\}$ be a sequence of positive numbers with $\sum_{n=1}^{\infty} r_{n}^{p}<\infty$ for some $p>0$, and let $\mathcal{D}_{n}$ be a class of $N_{n}$ balls (or cubes) of diameter $r_{n}$ in $\mathbb{R}^{d}$ with $\log N_{n}=O(1)\left|\log r_{n}\right|$. If, for every constant $T>0$, there exists a sequence $\left\{t_{n}\right\}$ of positive numbers and constants $K_{3}$ and $\delta>0$ such that

$$
\mathbb{P}^{x}\left\{\inf _{t_{n} \leq s \leq T}|X(s)-x| \leq r_{n}\right\} \leq K_{3} r_{n}^{\delta}, \quad \forall x \in \mathbb{R}^{d},
$$

then there exists a constant $K_{4}$, depending on $p$ and $\delta$ only, such that, with $\mathbb{P}^{x}$-probability one, for $n$ large enough, $X^{-1}(B) \cap[0, T]$ can be covered by at most $K_{4}$ intervals of length $t_{n}$, whenever $B \in \mathcal{D}_{n}$. 
Proof. Let $B \in \mathcal{D}_{n}$ and assume $B=B\left(z, \frac{r_{n}}{2}\right)$ for some $z \in \mathbb{R}^{d}$ since the diameter of $B$ is $r_{n}$. Let $\tau_{0}=0$ and, for any $k \geq 1$, define

$$
\tau_{k}=\inf \left\{t \geq \tau_{k-1}+t_{n},\left|X_{t}-z\right| \leq \frac{r_{n}}{2}\right\},
$$

with the convention inf $\varnothing=\infty$. It is easy to see

$$
\left\{t: X_{t} \in B\right\} \subseteq \bigcup_{i=0}^{\infty}\left[\tau_{i}, \tau_{i}+t_{n}\right)
$$

which implies

$$
\left\{\tau_{k}>T\right\} \subseteq\left\{X^{-1}(B, \omega) \cap[0, T] \text { can be covered by } k \text { intervals of length } t_{n}\right\} .
$$

Hence,

$$
\left\{X^{-1}(B, \omega) \cap[0, T] \text { cannot be covered by } k \text { intervals of length } t_{n}\right\} \subseteq\left\{\tau_{k} \leq T\right\} .
$$

By the strong Markov property, (2.4) and the fact that $X\left(\tau_{k-1}\right) \in B$ as $\tau_{k-1} \leq T$, we obtain

$$
\begin{aligned}
\mathbb{P}^{x}\left\{\tau_{k} \leq T\right\} & \leq \mathbb{P}^{x}\left\{\tau_{k} \leq T \mid \tau_{k-1} \leq T\right\} \mathbb{P}^{x}\left\{\tau_{k-1} \leq T\right\} \\
& \leq \sup _{y \in B} \mathbb{P}^{y}\left\{\inf _{t_{n} \leq s \leq T}|X(s)-z| \leq \frac{r_{n}}{2}\right\} \mathbb{P}^{x}\left\{\tau_{k-1} \leq T\right\} \\
& =\sup _{y \in B} \mathbb{P}^{y}\left\{\inf _{t_{n} \leq s<T}|X(s)-y+y-z| \leq \frac{r_{n}}{2}\right\} \mathbb{P}^{x}\left\{\tau_{k-1} \leq T\right\} \\
& \leq \sup _{y \in B} \mathbb{P}^{y}\left\{\inf _{t_{n} \leq s \leq T}|X(s)-y| \leq r_{n}\right\} \mathbb{P}^{x}\left\{\tau_{k-1} \leq T\right\} \\
& \leq K_{3} r_{n}^{\delta} \mathbb{P}^{x}\left\{\tau_{k-1} \leq T\right\} .
\end{aligned}
$$

By iterating the above argument, we obtain

$$
\mathbb{P}^{x}\left\{\tau_{k} \leq T\right\} \leq K_{3}^{k} r_{n}^{k \delta} .
$$

Define the events

$$
\begin{aligned}
A_{n}^{k}:= & \left\{\omega \in \Omega: \exists B \in \mathcal{D}_{n} \text { s.t. } X^{-1}(B, \omega) \cap[0, T]\right. \text { cannot be covered by } \\
& \left.k \text { intervals of length } t_{n}\right\} .
\end{aligned}
$$

Since $\log N_{n}=O(1)\left|\log r_{n}\right|$, i.e., there exists a positive constant $C$ such that $N_{n} \leq r_{n}^{-C}$ for all integers $n$, we see that for $k$ large enough (say, $\delta k-C \geq p$ ),

$$
\sum_{n=1}^{\infty} \mathbb{P}^{x}\left(A_{n}^{k}\right) \leq \sum_{n=1}^{\infty} N_{n} K_{3}^{k} r_{n}^{k \delta} \leq K_{3}^{k} \sum_{n=1}^{\infty} r_{n}^{-C+\delta k} \leq K_{3}^{k} \sum_{n=1}^{\infty} r_{n}^{p}<\infty .
$$

Hence, the conclusion of Lemma 2.2 follows from the Borel-Cantelli lemma. 


\section{Main result}

The objective of this section is to establish uniform Hausdorff and packing dimension results for the images of a time homogeneous Markov process $X=\left\{X(t), t \geq 0, \mathbb{P}^{x}\right\}$ with values in $\mathbb{R}^{d}$. For any Borel set $A$ in $\mathbb{R}^{d}$, denote by $P(t, x, A):=\mathbb{P}^{x}\left(X_{t} \in A\right)$ the transition probability of $X$. We state the following assumptions, where (A1) will be used for deriving uniform upper bounds, and (A2) for uniform lower bounds.

(A1) There is a constant $H>0$ such that for any $\gamma \in(0, H)$, there exist constants $C>0$, $\eta>0$ and $t_{0} \in(0,1)$ such that for all $x \in \mathbb{R}^{d}$ and $0<t \leq t_{0}$,

$$
\mathbb{P}^{x}\left\{\sup _{0 \leq s \leq t}|X(s)-x| \geq t^{\gamma}\right\} \leq C t^{\eta} .
$$

(A2) There is a sequence of vectors of non-negative numbers $J=\left\{\left(\varepsilon_{n}, \zeta_{n}\right), n \geq 1\right\}$ such that $\varepsilon_{n} \rightarrow 0$ and $\zeta_{n} \rightarrow 0$ as $n \rightarrow \infty$, and has the following property (for simplicity of notation, we omit the subscript $n$ ): For any $(\varepsilon, \zeta) \in J$ and constant $T>0$, there exist positive constants $C_{1}$, $C_{2}$, and $r_{0} \leq 1$ such that for all $0<r \leq r_{0}, x, y \in \mathbb{R}^{d}$ with $|y-x| \leq r$, and all $0<t \leq T$,

$$
P(t, y, B(x, r)) \geq C_{1} \min \left\{1,\left(\frac{r}{t^{H-\zeta}}\right)^{d+\varepsilon}\right\} ;
$$

and

$$
P(t, x, B(x, r)) \leq C_{2} \min \left\{1,\left(\frac{r}{t^{H+\zeta}}\right)^{d-\varepsilon}\right\} .
$$

(A3) We strengthen (A2) by further assuming that (3.3) holds for all $t>0$.

Condition (A2) is quite general due to the flexibility in choosing arbitrarily small constants $\varepsilon$ and $\zeta$, in order for (3.2) and (3.3) to hold. This condition can be satisfied by a large class of Markov processes such as those with a bounded transition density and an approximate scaling property; see Section 4 for some interesting examples. (A3) is slightly stronger than (A2), which is needed for our subordination argument in proving Theorem 3.3 below in the critical case of $1=H d$.

Condition (A1) is less obvious. In the following, we give a sufficient condition for it to hold. For any $h \geq 0$ and $a>0$, similar to Manstavičius [41], we consider the function

$$
\alpha(h, a)=\sup \left\{P\left(s, x, B(x, a)^{c}\right): x \in \mathbb{R}^{d}, 0 \leq s \leq h\right\},
$$

where $B(x, a)^{c}=\left\{y \in \mathbb{R}^{d}:|y-x| \geq a\right\}$. The function $h \mapsto \alpha(h, a)$ carries a lot of information about regularity properties of the sample paths of the Markov process $X$. For example, Kinney [30] showed that if for each fixed $a>0, \alpha(h, a) \rightarrow 0$ as $h \rightarrow 0$ then the sample function $X(t)$ is almost surely cadlag; and if $\alpha(h, a)=o(h)$ as $h \rightarrow 0$ for every fixed $a>0$ then the sample function $X(t)$ is almost surely continuous. See Manstavičius [41] and the references therein for further information. 
For a given constant $H>0$, a Markov process $X$ is said to belong to the class $\widetilde{\mathcal{M}}(H)$ if there exist positive and finite constants $C, \beta, h_{0}$ and $a_{0}$, depending on $d$ and $H$ only, such that the following property holds: For all $h \in\left(0, h_{0}\right)$ and $a \in\left(0, a_{0}\right)$ such that $h a^{-1 / H}<1$, we have

$$
\alpha(h, a) \leq C\left(\frac{h}{a^{1 / H}}\right)^{\beta} .
$$

Condition (3.5) is the same as (1.1) in Manstavičius [41] for the class $\mathcal{M}(\beta, \gamma)$ with $\gamma=\beta / H$. We mention that Schilling and Schnurr [51], Corollary 5.10, proved that the solution of certain SDE driven by a Lévy process belongs to the class $\mathcal{M}(1, \gamma)$ of Manstavičius [41] for suitable choice of $\gamma$, thus derived a result on the $\gamma$-variation of the solution.

The following sufficient condition for (A1) is often convenient to use (cf. Theorem 4.1 below).

Proposition 3.1. Let $X=\left\{X(t), t \in \mathbb{R}_{+}, \mathbb{P}^{x}\right\}$ be a separable, time-homogeneous Markov process taking values in $\mathbb{R}^{d}$. If $X$ belongs to the class $\widetilde{\mathcal{M}}(H)$, then for any $\varepsilon \in(0,1)$ and $\gamma \in(0, H)$, $X$ satisfies (3.1) with $\eta=\beta\left(1-\frac{\gamma}{H}\right)$.

Proof. We make use of the following Ottaviani-type inequality (cf. Gikhman and Skorohod [14], page 420, or Manstavičius [41]): For all $x \in \mathbb{R}^{d}$, all $h>0$ and $a>0$ such that $\alpha(h, a / 2)<1$,

$$
\mathbb{P}^{x}\left\{\sup _{0 \leq s \leq h}|X(s)-X(0)|>a\right\} \leq \frac{\mathbb{P}^{x}\{|X(h)-X(0)|>a / 2\}}{1-\alpha(h, a / 2)} .
$$

For any $\gamma \in(0, H)$, it follows from (3.6) with $a=h^{\gamma}$ and (3.5) that for $h$ small enough,

$$
\mathbb{P}^{x}\left\{\sup _{0 \leq s \leq h}|X(s)-X(0)|>h^{\gamma}\right\} \leq C \mathbb{P}^{x}\left\{|X(h)-X(0)|>h^{\gamma} / 2\right\} \leq C h^{\beta\left(1-\frac{\gamma}{H}\right)} .
$$

This proves the proposition.

Most examples given in Section 4 are Lévy or Lévy-type processes. For these processes, the maximal tail probability $\mathbb{P}^{x}\left\{\sup _{s \in[0, t]}\left|X_{s}-x\right| \geq r\right\}$ in (3.7) has been studied by several authors. Pruitt [46] established an upper bound for the maximal probability for a general Lévy process in terms of its Lévy measure. Schilling [49] and Böttcher, Schilling and Wang [8] extended Pruitt's result to Lévy-type processes and proved an upper bound in terms of the symbol of the process. The following proposition is taken from Böttcher, Schilling and Wang [8], Corollary 5.2, which can be applied to verify (A1) for Lévy-type processes. We remark that Kühn [37], Lemma 3.2 has proved recently that the inequality (3.8) still holds if $t$ is a stopping time, with the $t$ on the right-hand side replaced by $\mathbb{E}(t)$. We thank the referee for pointing out these results to us.

Proposition 3.2. Let $X=\{X(t), t \geq 0\}$ be a Lévy-type process with a symbol $q(x, \xi): \mathbb{R}^{d} \times$ $\mathbb{R}^{d} \rightarrow \mathbb{C}$ given by

$$
q(x, \xi)=-i b(x) \cdot \xi+\frac{1}{2} \xi \cdot Q(x) \xi+\int_{\mathbb{R}^{d} \backslash\{0\}}\left(1-e^{i y \cdot \xi}+i y \cdot \xi 1_{(0,1]}(|y|)\right) v(x, d y),
$$


where for each fixed $x \in \mathbb{R}^{d},(b(x), Q(x), v(x, d y))$ is a Lévy triplet, i.e. $b(x) \in \mathbb{R}^{d}, Q(x) \in$ $\mathbb{R}^{d \times d}$ is a symmetric positive semidefinite matrix and $v(x, d y)$ is a measure on $\left(\mathbb{R}^{d} \backslash\{0\}, \mathcal{B}\left(\mathbb{R}^{d} \backslash\right.\right.$ $\{0\}))$ such that $\int_{\mathbb{R}^{d} \backslash\{0\}}\left(|y|^{2} \wedge 1\right) v(x, d y)<\infty$. Then, there exists a constant $C>0$ such that

$$
\mathbb{P}^{x}\left\{\sup _{s \in[0, t]}\left|X_{s}-x\right| \geq r\right\} \leq C t \sup _{|y-x| \leq r} \sup _{|\xi| \leq 1 / r}|q(y, \xi)| .
$$

Our main theorem of this paper is the following uniform Hausdorff and packing dimension result for the images of $X$.

Theorem 3.3. Let $X=\left\{X(t), t \in \mathbb{R}_{+}, \mathbb{P}^{x}\right\}$ be a time homogeneous Markov process in $\mathbb{R}^{d}$ and satisfies Conditions (A1). Assume either (i) $1<H d$ and (A2) hold; or (ii) $1=H d$ and (A3) hold. Then for all $x \in \mathbb{R}^{d}$,

$$
\mathbb{P}^{x}\left\{\operatorname{dim}_{\mathrm{H}} X(E)=\frac{1}{H} \operatorname{dim}_{\mathrm{H}} E \text { for all Borel sets } E \subseteq[0, \infty)\right\}=1
$$

and

$$
\mathbb{P}^{x}\left\{\operatorname{dim}_{P} X(E)=\frac{1}{H} \operatorname{dim}_{P} E \text { for all Borel sets } E \subseteq[0, \infty)\right\}=1 .
$$

Proof. We will only prove the Hausdorff dimension result (3.9). The proof of (3.10), which is based on the connection between packing dimension and upper box-counting dimension (cf. Falconer [13]), is similar and hence omitted.

The proof of is divided into two parts. Namely, we prove the upper and lower bounds for $\operatorname{dim}_{\mathrm{H}} X(E)$, respectively.

Part 1 (Uniform upper bound). By the $\sigma$-stability of Hausdorff dimension (cf. Falconer [13]), it suffices to consider Borel sets $E \subseteq[0, L]$ for all fixed integers $L$. For simplicity, we take $L=1$ in this proof. Let $\gamma \in(0, H)$ be a constant, $t_{n}=2^{-n}$ and

$$
\mathcal{C}_{n}=\left\{\left[(j-1) t_{n}, j t_{n}\right]: j=1,2, \ldots, 2^{n}\right\} .
$$

By Condition (A1), we get that for all $x \in \mathbb{R}^{d}$,

$$
\mathbb{P}^{x}\left\{\sup _{0 \leq s \leq t_{n}}|X(s)-x| \geq t_{n}^{\gamma}\right\} \leq C t_{n}^{\eta} .
$$

Thus, by Lemma 2.1, with probability one under $\mathbb{P}^{x}$, as $n$ is sufficiently large, $X(I)$ can be covered by $K_{2}$ balls of radius $\theta_{n}:=t_{n}^{\gamma}$ for all intervals $I \in \mathcal{C}_{n}$.

Let $\chi=\operatorname{dim}_{H} E$. Then, for any $\delta>0$, there exists a sequence of intervals $\left\{F_{i} \subseteq[0,1], i \in \mathbb{N}\right\}$ such that $d_{i}:=\operatorname{diam}\left(F_{i}\right) \leq \varepsilon$,

$$
E \subseteq \bigcup_{i} F_{i} \text { and } \sum_{i} d_{i}^{\chi+\delta} \leq 1
$$

We choose $n_{i}$ so that $\frac{t_{n_{i}}}{2} \leq d_{i} \leq t_{n_{i}}$, thus $F_{i}$ is contained in at most two intervals of $\mathcal{C}_{n_{i}}$. Consequently, $X\left(F_{i}\right)$ can be covered by $2 K_{2}$ balls of radius $\theta_{n_{i}}$, which are denoted by $B_{i, 1}, \ldots, B_{i, 2 K_{2}}$. 
Hence,

$$
X(E) \subseteq \bigcup_{i}^{2 K_{2}} \bigcup_{j=1} B_{i, j}
$$

Further observe that, by using (3.11), we have

$$
\begin{aligned}
\sum_{i} \sum_{j=1}^{2 K_{2}}\left[\operatorname{diam}\left(B_{i, j}\right)\right]^{(\chi+\delta) / \gamma} & \leq 2 K_{2} \sum_{i} t_{n_{i}}^{\chi+\delta} \\
& \leq 2 K_{2} \sum_{i}\left(2 d_{i}\right)^{\chi+\delta} \leq 2^{1+\chi+\delta} K_{2} .
\end{aligned}
$$

This yields $\operatorname{dim}_{\mathrm{H}} X(E) \leq(\chi+\delta) / \gamma$. Letting $\delta \downarrow 0$ and $\gamma \uparrow H$ yields

$$
\operatorname{dim}_{\mathrm{H}} X(E) \leq \frac{1}{H} \operatorname{dim}_{\mathrm{H}} E .
$$

Part 2 (Uniform lower bound). In order to prove

$$
\mathbb{P}^{x}\left\{\operatorname{dim}_{\mathrm{H}} X(E) \geq \frac{1}{H} \operatorname{dim}_{\mathrm{H}} E \text { for all Borel sets } E \subseteq[0, \infty)\right\}=1,
$$

we will treat the two cases (i) $1<H d$ and (ii) $1=H d$ separately.

We observe that the inequality in (3.12) follows from the following claim: For all $x \in \mathbb{R}^{d}$,

$$
\mathbb{P}^{x}\left\{\operatorname{dim}_{\mathrm{H}} X^{-1}(F) \leq H \operatorname{dim}_{\mathrm{H}} F \text { for all } F \subseteq \mathbb{R}^{d}\right\}=1,
$$

by taking $F=X(E)$. Moreover, by the $\sigma$-stability of Hausdorff dimension, (3.13) is equivalent to: For all constants $T>0$ and all integers $m=1,2, \ldots$,

$$
\mathbb{P}^{x}\left\{\operatorname{dim}_{\mathrm{H}}\left(X^{-1}(F) \cap[0, T]\right) \leq H \operatorname{dim}_{\mathrm{H}} F \text { for all } F \subseteq[-m, m]^{d}\right\}=1 .
$$

Hence, in order to prove (3.12) for the case (i), it suffices to prove (3.14) for all fixed constant $T>0$ and positive integer $m$. This will be done by using the covering principle in Lemma 2.2. Recall that in case (i) we assume (A2) holds. Specifically, (3.2) and (3.3) hold for all $0<t \leq 2 T$.

By applying Xiao [55], Proposition 2.1, which is an extension of Theorem 1.1 in Khoshnevisan [23], we get

$$
\mathbb{P}^{x}\left\{\inf _{t \leq s \leq T}|X(s)-x| \leq r\right\} \leq \frac{\int_{t}^{2 T} P(s, x, B(x, r)) d s}{\inf _{|y-x| \leq r} \int_{0}^{T-t} P(s, y, B(x, r)) d s} .
$$

In order to estimate the denominator of (3.15), we assume without loss of generality that $0<t \leq$ $T / 2$. For any $y \in \mathbb{R}^{d}$ with $|y-x| \leq r \leq r_{0}$, we use (3.2) in (A2) to get

$$
\int_{0}^{T-t} P(s, y, B(x, r)) d s \geq C_{1} \int_{0}^{T / 2} \min \left\{1,\left(\frac{r}{s^{H-\zeta}}\right)^{d+\varepsilon}\right\} d s \geq C r^{\frac{1}{H-\zeta}} .
$$


On the other hand, since $1<H d$, we choose $(\varepsilon, \zeta) \in J$ such that $\varepsilon$ and $\zeta$ are small enough so that $1<(H+\zeta)(d-\varepsilon)$. By using (3.3) in (A2), we derive that for any $t>r^{1 /(H+\zeta)}$,

$$
\begin{aligned}
\int_{t}^{2 T} P(s, x, B(x, r)) d s & \leq C_{2} \int_{t}^{2 T} \min \left\{1,\left(\frac{r}{s^{H+\zeta}}\right)^{d-\varepsilon}\right\} d s \\
& =C_{2} \int_{t}^{2 T}\left(\frac{r}{s^{H+\zeta}}\right)^{d-\varepsilon} d s \leq C r^{d-\varepsilon} t^{1-(H+\zeta)(d-\varepsilon)} .
\end{aligned}
$$

We combine the above with (3.15) to see that for all $x \in \mathbb{R}^{d}, r>0$ and $t>r^{1 /(H+\zeta)}$,

$$
\mathbb{P}^{x}\left\{\inf _{t \leq s \leq T}|X(s)-x| \leq r\right\} \leq C r^{d-\frac{1}{H-\zeta}-\varepsilon} t^{1-(H+\zeta)(d-\varepsilon)}
$$

Now we proceed to prove (3.14) for the case $1<H d$. Fix any integer $m \geq 1$ and let $\mathcal{D}_{n}^{m}$ be the collection of dyadic cubes in $[-m, m]^{d}$ of the form $\prod_{i=1}^{d}\left[j_{i} 2^{-n},\left(j_{i}+1\right) 2^{-n}\right]$, where each $j_{i}$ is an integer and $j_{i} \in\left[-m 2^{n}, m 2^{n}-1\right]$. It is easy to see that $\sharp\left(\mathcal{D}_{n}^{m}\right)=\left(m 2^{n+1}\right)^{d}$.

Fix a constant $\gamma \in(0,1 / H)$ and then choose $\varepsilon>0$ and $\zeta>0$ sufficiently small such that $(\varepsilon, \zeta) \in J$ and

$$
\delta:=\left(d-\frac{1}{H-\zeta}-\varepsilon\right)(1-\gamma(H+\zeta))-\frac{2 \gamma \zeta}{H-\zeta}>0 .
$$

This is possible since $\left(d-\frac{1}{H}\right)(1-\gamma H)>0$.

Let $r_{n}=2^{-n}$ and $t_{n}=2^{-\gamma n}$, further choose $n$ large enough so that $r_{n} \leq r_{0}$. Notice that $t_{n}>$ $r_{n}^{1 /(H+\zeta)}$. By (3.16) we verify that for all $x \in \mathbb{R}^{d}$,

$$
\mathbb{P}^{x}\left\{\inf _{t_{n} \leq s \leq T}\left|X_{S}-x\right| \leq r_{n}\right\} \leq C r_{n}^{d-\frac{1}{H-\zeta}-\varepsilon} t_{n}^{1-(H+\zeta)(d-\varepsilon)}=C r_{n}^{\delta}
$$

Hence, Lemma 2.2 implies that there is an integer $K_{4}$ such that with $\mathbb{P}^{x}$-probability one, for all $n$ large enough (say, $n \geq n_{0}$ ) and all dyadic cubes $B \in \mathcal{D}_{n}^{m}, X^{-1}(B) \cap[0, T]$ can be covered by at most $K_{4}$ intervals of length $t_{n}=2^{-\gamma n}$.

For any Borel set $F \subseteq[-m, m]^{d}$, let $\theta>\operatorname{dim}_{\mathrm{H}} F$. Then there exists a sequence of dyadic cubes $\left\{B_{i}, i \in \mathbb{N}\right\}$ in $[-m, m]^{d}$ of sides $r_{n_{i}}=2^{-n_{i}}$ such that $n_{i} \geq n_{0}$,

$$
F \subseteq \bigcup_{i=1}^{\infty} B_{i} \quad \text { and } \quad \sum_{i=1}^{\infty} r_{n_{i}}^{\theta} \leq 1
$$

Since, for every $i, X^{-1}\left(B_{i}\right) \cap[0, T]$ can be covered by $K_{4}$ intervals $I_{i j}$ of length $t_{n_{i}}=2^{-\gamma n_{i}}$, we see that

$$
X^{-1}(F) \cap[0, T] \subseteq \bigcup_{i=1}^{\infty} \bigcup_{j=1}^{K_{4}} I_{i j}
$$


Moreover,

$$
\sum_{i=1}^{\infty} \sum_{j=1}^{K_{4}}\left[\operatorname{diam}\left(I_{i j}\right)\right]^{\theta / \gamma} \leq K_{4} \sum_{i=1}^{\infty} r_{n_{i}}^{\theta} \leq K_{4},
$$

this implies $\operatorname{dim}_{\mathrm{H}}\left(X^{-1}(F) \cap[0, T]\right) \leq \frac{\theta}{\gamma}$. Letting $\gamma \uparrow 1 / H$ and $\theta \downarrow \operatorname{dim}_{\mathrm{H}} F$ yields (3.14) and, thus, (3.12) for the case $1<H d$.

Finally, we prove (3.12) for the case $1=H d$ by making use of a "subordination argument" that is similar to that in Hawkes [16] (see also Pruitt [45]).

Let $\tau=\left\{\tau_{t}, t \geq 0\right\}$ be a stable subordinator with stability index $\rho \in(0,1)$, and independent of the process $X$. Consider the Markov process $Y=\left\{Y_{t}, t \geq 0\right\}$ defined by $Y_{t}=X\left(\tau_{t}\right)$. It is easy to see $\left\{Y_{t}, t \geq 0\right\}$ is a time-homogeneous strong Markov process. Denote the transition probability of $Y$ by $\tilde{P}(t, x, A):=\mathbb{P}^{x}\left(Y_{t} \in A\right)$. We claim that, if (A3) holds, then $\tilde{P}(t, x, A)$ satisfies Condition (A2) with $H$ replaced by $H / \rho$. Consequently, because $1<H d / \rho$, we can apply the conclusion of the first part to the process $Y$.

More specifically, we now verify the following claim under assumption (A3):

(A2') We can find a sequence $J^{\prime}=\left\{\left(\varepsilon^{\prime}, \zeta^{\prime}\right)\right\}$ of arbitrarily small numbers with the following property: For any $\left(\varepsilon^{\prime}, \zeta^{\prime}\right) \in J^{\prime}$ and $A>0$, there exist positive constants $C_{3}$ and $C_{4}$ such that for all $0<r \leq r_{0}$ (as in (A2)), $x, y \in \mathbb{R}^{d}$ with $|y-x| \leq r$ and $0<t \leq A$,

$$
\tilde{P}(t, y, B(x, r)) \geq C_{3} \min \left\{1,\left(\frac{r}{t^{\left(H-\zeta^{\prime}\right) / \rho}}\right)^{d+\varepsilon^{\prime}}\right\} ;
$$

and

$$
\tilde{P}(t, x, B(x, r)) \leq C_{4} \min \left\{1,\left(\frac{r}{t^{\left(H+\zeta^{\prime}\right) / \rho}}\right)^{d-\varepsilon^{\prime}}\right\} .
$$

To verify $\left(\mathrm{A} 2^{\prime}\right.$ ), we denote, for all $t>0$, the density function of $\tau_{t}$ by $p_{\tau_{t}}$. Then the selfsimilarity of $\tau_{t}$ implies $p_{\tau_{t}}(s)=t^{-\frac{1}{\rho}} p_{\tau_{1}}\left(t^{-\frac{1}{\rho}} s\right)$. Hence,

$$
\begin{aligned}
\tilde{P}(t, y, B(x, r)) & =\mathbb{P}^{y}\left(\left|Y_{t}-x\right| \leq r\right) \\
& =\int_{0}^{\infty} \mathbb{P}^{y}\left(\left|X_{\tau_{t}}-x\right| \leq r \mid \tau_{t}=s\right) p_{\tau_{t}}(s) d s \\
& =\int_{0}^{\infty} P(s, y, B(x, r)) p_{\tau_{t}}(s) d s \\
& =\int_{0}^{\infty} P(s, y, B(x, r)) t^{-\frac{1}{\rho}} p_{\tau_{1}}\left(t^{-\frac{1}{\rho}} s\right) d s \\
& =\int_{0}^{\infty} P\left(t^{\frac{1}{\rho}} s, y, B(x, r)\right) p_{\tau_{1}}(s) d s .
\end{aligned}
$$

Consequently, we can make use of Condition (A3) to estimate $\tilde{P}(t, y, B(x, r))$. Recall that $J=$ $\{(\varepsilon, \zeta)\}$ is the sequence in (A2). 
On one hand, for any constant $A>0$, we apply (3.2) with $T \geq 2 A^{1 / \rho}$ to derive that for any $x, y \in \mathbb{R}^{d}$ with $|y-x| \leq r, 0<r \leq r_{0}$ and $0<t \leq A$, we have

$$
\begin{aligned}
\tilde{P}(t, y, B(x, r)) \geq & C_{1} \int_{0}^{T t^{-1 / \rho}} \min \left\{1,\left(\frac{r}{\left(t^{1 / \rho} s\right)^{H-\zeta}}\right)^{d+\varepsilon}\right\} p_{\tau_{1}}(s) d s \\
= & C_{1} \int_{0}^{r^{1 /(H-\zeta)} t^{-1 / \rho}} p_{\tau_{1}}(s) d s \\
& +C_{1} \int_{r^{1 /(H-\zeta)} t^{-1 / \rho}}^{T t^{-1 / \rho}}\left(\frac{r}{t^{(H-\zeta) / \rho}}\right)^{d+\varepsilon} \frac{p_{\tau_{1}}(s)}{s^{(H-\zeta)(d+\varepsilon)}} d s .
\end{aligned}
$$

It is easy to see that when $r^{1 /(H-\zeta)}>t^{1 / \rho}$,

$$
\tilde{P}(t, y, B(x, r)) \geq C_{1} \int_{0}^{1} p_{\tau_{1}}(s) d s
$$

and when $r^{1 /(H-\zeta)} \leq t^{1 / \rho}$,

$$
\tilde{P}(t, y, B(x, r)) \geq C_{1}\left(\frac{r}{t^{(H-\zeta) / \rho}}\right)^{d+\varepsilon} \int_{1}^{2} \frac{1}{s^{(H-\zeta)(d+\varepsilon)}} p_{\tau_{1}}(s) d s,
$$

where we have used that fact that $T t^{-1 / \rho} \geq T A^{-1 / \rho} \geq 2$ for all $0<t \leq A$ and the last integral is positive since the density $p_{\tau_{1}}(s)$ is positive for $s>0$. Then, (3.22) and (3.23) imply (3.19) with $\varepsilon^{\prime}=\varepsilon$ and $\zeta^{\prime}=\zeta$.

On the other hand, similarly to (3.21), we use (3.3), which is now assumed to hold for all $t>0$, to derive that for any $x \in \mathbb{R}^{d}, 0<r \leq r_{0}$ and $t>0$,

$$
\begin{aligned}
\tilde{P}(t, x, B(x, r)) \leq & C_{2} \int_{0}^{\infty} \min \left\{1,\left(\frac{r}{\left(t^{1 / \rho} s\right)^{H+\zeta}}\right)^{d-\varepsilon}\right\} p_{\tau_{1}}(s) d s \\
= & C_{2} \int_{0}^{r^{1 /(H+\zeta)} t^{-1 / \rho}} p_{\tau_{1}}(s) d s \\
& +C_{2} \int_{r^{1 /(H+\zeta)} t^{-1 / \rho}}^{\infty}\left(\frac{r}{t^{(H+\zeta) / \rho}}\right)^{d-\varepsilon} \frac{p_{\tau_{1}}(s)}{s^{(H+\zeta)(d-\varepsilon)}} d s .
\end{aligned}
$$

Since $\tilde{P}(t, y, B(x, r)) \leq 1$, in order to verify (3.20), we only need to consider the case when $r^{1 /(H+\zeta)} \leq t^{1 / \rho}$. If $(H+\zeta)(d-\varepsilon)<1$, then by the boundedness of the density function $p_{\tau_{1}}(\cdot)$, i.e., $\sup _{0<s \leq 1} p_{\tau_{1}}(s) \leq M$ for some $M>0$, we obtain that

$$
\tilde{P}(t, y, B(x, r)) \leq C_{2} M \frac{r^{1 /(H+\zeta)}}{t^{1 / \rho}}+C_{2}\left(\frac{r}{t^{(H+\zeta) / \rho}}\right)^{d-\varepsilon} \int_{0}^{\infty} \frac{p_{\tau_{1}}(s)}{s^{(H+\zeta)(d-\varepsilon)}} d s .
$$

The last integral is convergent at 0 because $(H+\zeta)(d-\varepsilon)<1$ and at infinity because $p_{\tau_{1}}(s) \sim$ $\frac{C_{\rho}}{s^{1+\rho}}$ as $s \rightarrow+\infty$. 
If $(H+\zeta)(d-\varepsilon)>1$, then we further split the last integral in (3.24) over $\left[r^{1 /(H+\zeta)} t^{-1 / \rho}, 1\right]$ and $[1, \infty)$, and to derive

$$
\tilde{P}(t, y, B(x, r)) \leq C_{2}^{\prime} M \frac{r^{1 /(H+\zeta)}}{t^{1 / \rho}}+C_{2}\left(\frac{r}{t^{(H+\zeta) / \rho}}\right)^{d-\varepsilon} \int_{1}^{\infty} \frac{p_{\tau_{1}}(s)}{s^{(H+\zeta)(d-\varepsilon)}} d s,
$$

where $C_{2}^{\prime}$ is a finite constant and, again, the last integral is convergent. The case of $(H+\zeta)(d-$ $\varepsilon)=1$ can be treated in the same way, and (3.26) still holds with an extra factor of $\log \left(\frac{r^{1 /(H+\zeta)}}{t^{1 / \rho}}\right)$ in the first term on the right-hand side, which can be absorbed by choosing $\tilde{\varepsilon}$ below slightly bigger. However, for simplicity, we ignore this case because one may choose $J=\{(\varepsilon, \zeta)\}$ so that $(H+\zeta)(d-\varepsilon) \neq 1$ for all $(\varepsilon, \zeta) \in J$.

Notice that, when $1=H d$, we can write $\frac{1}{H+\zeta}=d-\tilde{\varepsilon}$, where $\tilde{\varepsilon}=\frac{\zeta}{H(H+\zeta)}$. It follows from (3.25) and (3.26) that for $r^{1 /(H+\zeta)} \leq t^{1 / \rho}$,

$$
\tilde{P}(t, y, B(x, r)) \leq C\left(\frac{r}{t^{(H+\zeta) / \rho}}\right)^{d-\varepsilon^{\prime}}
$$

for some finite constant $C$, where $\varepsilon^{\prime}=\max \{\varepsilon, \tilde{\varepsilon}\}$. Thus, we have verified (3.20) with $\varepsilon^{\prime}=$ $\max \{\varepsilon, \tilde{\varepsilon}\}$ and $\zeta^{\prime}=\zeta$. Moreover, because $0<t \leq A$, the inequality in (3.19) remains valid (with a modified constant $C_{3}$ ) if we take $\varepsilon^{\prime}=\max \{\varepsilon, \tilde{\varepsilon}\}$. Thus, Condition (A2') has been verified.

Because of Condition $\left(\mathrm{A} 2^{\prime}\right)$ and the fact that $\frac{H d}{\rho}>1$, we can apply the above uniform lower bound result in the case of $H d>1$ to Markov process $Y$ to obtain

$$
\mathbb{P}\left\{\operatorname{dim}_{\mathrm{H}} Y(E) \geq \frac{\rho}{H} \operatorname{dim}_{\mathrm{H}} E \text { for all Borel sets } E \subseteq[0, \infty)\right\}=1 .
$$

It follows that, with probability 1 , for all Borel sets $E \subseteq[0, \infty)$,

$$
\operatorname{dim}_{\mathrm{H}} X(E) \geq \operatorname{dim}_{\mathrm{H}} X(\tau(B))=\operatorname{dim}_{\mathrm{H}} Y(B) \geq \frac{\rho}{H} \operatorname{dim}_{\mathrm{H}} B,
$$

where $B=\left\{t: \tau_{t} \in E\right\}=\tau^{-1}(E)$. Even though both $\operatorname{dim}_{\mathrm{H}} X(E)$ and $\operatorname{dim}_{\mathrm{H}} B$ in (3.28) are random, they are determined by two independent processes $X$ and $\tau$, respectively. Hence, we have

$$
\mathbb{P}\left\{\operatorname{dim}_{\mathrm{H}} X(E) \geq \frac{\rho}{H}\left\|\operatorname{dim}_{\mathrm{H}} \tau^{-1}(E)\right\|_{\infty} \text { for all Borel sets } E \subseteq[0, \infty)\right\}=1,
$$

where $\|\cdot\|_{\infty}$ is the $L^{\infty}(\mathbb{P})$-norm in the underlying probability space. (A more illustrative way for deriving (3.29) is to use the setting of product probability space. Namely, we assume that $X$ is defined on $\Omega, \tau$ is defined on $\Omega^{\prime}$, then $Y$ is defined on $\Omega \times \Omega^{\prime}$ and (3.28) holds for almost all $\left(\omega, \omega^{\prime}\right)$. One can see that (3.29) follows from (3.28) and Fubini's theorem.)

Recalling from Hawkes [17] that

$$
\left\|\operatorname{dim}_{H} \tau^{-1}(E)\right\|_{\infty}=\frac{\rho+\operatorname{dim}_{H} E-1}{\rho},
$$


we derive that

$$
\mathbb{P}\left\{\operatorname{dim}_{\mathrm{H}} X(E) \geq \frac{\rho+\operatorname{dim}_{\mathrm{H}} E-1}{H} \text { for all Borel sets } E \subseteq[0, \infty)\right\}=1 .
$$

Letting $\rho \uparrow 1$ yields (3.12) for the case $1=H d$. This completes the proof of Theorem 3.3.

\section{Examples}

Theorem 3.3 is applicable to a wide class of Markov processes. In this section, we provide some examples which include self-similar Markov processes, Lévy processes, stable jump diffusion processes and non-symmetric stable-type processes.

\subsection{Self-similar Markov processes}

The class of $H$-self-similar ( $H$-s.s.) Markov processes with values in $[0, \infty)$ was introduced and studied by Lamperti [39], who used the term "semi-stable" instead of "self-similar". The $H$-s.s. Markov processes on $\mathbb{R}^{d}$ or $\mathbb{R}^{d} \backslash\{0\}$ were investigated by, in chronicle order, Kiu [31], Graversen and Vuolle-Apiala [15], Vuolle-Apiala and Graversen [54], Vuolle-Apiala [53], Liu and Xiao [40], Xiao [55], Bertoin and Yor [6], Chaumont, Pantí and Rivero [9], Alili et al. [1], among others.

We recall the definition of $H$-self-similar processes. Let $(E, \mathcal{B})$ denotes $\mathbb{R}^{d}, \mathbb{R}^{d} \backslash\{0\}$ or $\mathbb{R}_{+}^{d}$ with the usual Borel $\sigma$ - algebra, $\{e\}$ is a point attached to $E$ as an isolated point. $\Omega$ denotes the space of all functions $\omega$ from $[0, \infty)$ to $E \cup\{e\}$ having the following properties:

(i) $\omega(t)=e$ for $t \geq \tau$, where $\tau=\inf \{t \geq 0: \omega(t)=e\}$;

(ii) $\omega$ is right continuous and has a left limit at every $t \in[0, \infty)$.

Let $H>0$ be a fixed constant. A time homogeneous Markov process $X=\left\{X(t), t \geq 0, \mathbb{P}^{x}\right\}$ with state space $E \cup\{e\}$ is called $H$-self-similar ( $H$-s.s.) if its transition probability function $P(t, x, A)$ satisfies

$$
P(t, x, A)=P\left(r t, r^{H} x, r^{H} A\right), \quad \text { for all } t>0, r>0, x \in E, A \in \mathcal{B} .
$$

The constant $H$ is called the self-similarity index of $X$. Condition (4.1) is equivalent to the statement that for every constant $r>0$ the $\mathbb{P}^{x}$-distribution of $\{X(t), t \geq 0\}$ is equal to the $\mathbb{P}^{H^{H} x_{-}}$ distribution of $\left\{r^{-H} X(r t), t \geq 0\right\}$. Important examples of self-similar Markov processes include strictly $\alpha$-stable Lévy processes which are $1 / \alpha$-s.s., the Bessel processes which form exactly the class of $1 / 2$-s.s. diffusions on $(0, \infty)$ (see Revuz and Yor [47]). More examples of $H$-s.s. Markov processes can be found in Lamperti [39], Graversen and Vuolle-Apiala [15], Kiu [31], Vuolle-Apiala and Graversen [54], Xiao [55].

In this section, we take $E=\mathbb{R}^{d}$ and assume the following two conditions:

(B1) There exist positive constants $\beta, C$ and $a_{1}$ such that

$$
P\left(1, x, B(x, a)^{c}\right) \leq C a^{-\beta} \quad \text { for all } x \in \mathbb{R}^{d} \text { and } a>a_{1} .
$$


(B2) For any $\varepsilon>0$ small, there exist positive constants $C_{1}$ and $C_{2}$ such that for all $r>0$ and $x, y \in \mathbb{R}^{d}$ with $|x-y| \leq r$, we have

$$
C_{1} \min \left\{1, r^{d+\varepsilon}\right\} \leq P(1, x, B(y, r)) \leq C_{2} \min \left\{1, r^{d-\varepsilon}\right\} .
$$

The following theorem provides a uniform version for the Hausdorff dimension result in Liu and Xiao [40].

Theorem 4.1. Let $X=\left\{X(t), t \in \mathbb{R}_{+}\right\}$be an $H$-s.s. Markov process in $\mathbb{R}^{d}$ that satisfies conditions (B1) and (B2). If $1 \leq H d$, then for all $x \in \mathbb{R}^{d}$, with $\mathbb{P}^{x}$-probability one,

$$
\operatorname{dim}_{\mathrm{H}} X(E)=\frac{1}{H} \operatorname{dim}_{\mathrm{H}} E \quad \text { and } \quad \operatorname{dim}_{\mathrm{P}} X(E)=\frac{1}{H} \operatorname{dim}_{\mathrm{P}} E
$$

for all Borel sets $E \subseteq \mathbb{R}_{+}$.

Proof. It is sufficient to verify that Conditions (A1) and (A3) are satisfied. It follows from (4.1) and (4.2) that for any $t>0, x \in \mathbb{R}^{d}$ and $a>0$,

$$
P\left(t, x, B(x, a)^{c}\right)=P\left(1, x t^{-H}, B\left(x t^{-H}, a t^{-H}\right)^{c}\right) \leq C\left(a t^{-H}\right)^{-\beta}
$$

provided $a t^{-H} \geq a_{1}$. This implies that $\alpha(h, a) \leq C\left(h a^{-1 / H}\right)^{H \beta}$ for all $h, a>0$ satisfying $h a^{-1 / H} \leq a_{1}^{-1 / H}$. Hence, $X$ belongs to the class $\widetilde{\mathcal{M}}(H)$. It follows from Proposition 3.1 that Condition (A1) is satisfied. For verifying (A3), we apply (4.1) again, together with the first inequality in (4.3), to see that for all $t, r>0$ and $x, y \in \mathbb{R}^{d}$ with $|x-y| \leq r$,

$$
P(t, x, B(y, r))=P\left(1, x t^{-H}, B\left(y t^{-H}, r t^{-H}\right)\right) \geq C_{1} \min \left\{1,\left(\frac{r}{t^{H}}\right)^{d+\varepsilon}\right\} .
$$

Similarly, we have

$$
P(t, x, B(x, r))=P\left(1, x t^{-H}, B\left(x t^{-H}, r t^{-H}\right)\right) \leq C_{2} \min \left\{1,\left(\frac{r}{t^{H}}\right)^{d-\varepsilon}\right\} .
$$

Thus, Condition (A3) is satisfied with $J=\left\{\left(\varepsilon_{n}, 0\right)\right\}$, where $\varepsilon_{n} \downarrow 0$ can be taken arbitrarily. Therefore the conclusion of Theorem 4.1 follows from Theorem 3.3.

\subsection{Lévy processes}

A stochastic process $X=\{X(t), t \geq 0\}$ on a probability space $(\Omega, \mathcal{F}, \mathbb{P})$, with values in $\mathbb{R}^{d}$, is called a Lévy process, if $X$ has stationary and independent increments, $t \mapsto X(t)$ is continuous in probability and $\mathbb{P}\{X(0)=0\}=1$. It is well known that for $t \geq 0$, the characteristic function of $X(t)$ is given by

$$
\mathbb{E}\left[e^{i\langle\xi, X(t)\rangle}\right]=e^{-t \psi(\xi)},
$$


where, by the Lévy-Khintchine formula,

$$
\psi(\xi)=i\langle\mathrm{a}, \xi\rangle+\frac{1}{2}\left\langle\xi, \Sigma \xi^{\prime}\right\rangle+\int_{\mathbb{R}^{d}}\left[1-e^{i\langle x, \xi\rangle}+\frac{i\langle x, \xi\rangle}{1+|x|^{2}}\right] \mathrm{L}(d x), \quad \forall \xi \in \mathbb{R}^{d},
$$

and $a \in \mathbb{R}^{d}$ is fixed, $\Sigma$ is a non-negative definite, symmetric, $(d \times d)$ matrix, and $\mathrm{L}$ is a Borel measure on $\mathbb{R}^{d} \backslash\{0\}$ that satisfies

$$
\int_{\mathbb{R}^{d}} \frac{|x|^{2}}{1+|x|^{2}} \mathrm{~L}(d x)<\infty .
$$

The function $\psi$ is called the characteristic or Lévy exponent of $X$, and $\mathrm{L}$ is the corresponding Lévy measure. The characteristic exponent $\psi$ plays very important roles in studying the Lévy process $X$ and many sample path properties of $X$ can be described in terms of $\psi$. We also note that

$$
\Re \psi(\xi) \geq 0, \quad \text { and } \quad \Re \psi(-\xi)=\Re \psi(\xi), \quad \forall \xi \in \mathbb{R}^{d} .
$$

Notice that, if $X$ is symmetric (i.e., $X$ and $-X$ have the same law), then its Lévy exponent $\psi(\xi)$ is a nonnegative function.

A Lévy process $X$ in $\mathbb{R}^{d}$ is called a stable Lévy process with index $\alpha \in(0,2]$ if its Lévy measure $L$ is of the form

$$
\mathrm{L}(d x)=\frac{d r}{r^{1+\alpha}} v(d y), \quad \forall x=r y,(r, y) \in \mathbb{R}_{+} \times \mathbb{S}_{d},
$$

where $\mathbb{S}_{d}=\left\{y \in \mathbb{R}^{d}:|y|=1\right\}$ is the unit sphere in $\mathbb{R}^{d}$ and $v(d y)$ is an arbitrary finite Borel measure on $\mathbb{S}_{d}$. Stable Lévy processes in $\mathbb{R}^{d}$ of index $\alpha=1$ are also called Cauchy processes. It follows from (4.6) and (4.7) that the Lévy exponent $\psi_{\alpha}$ of a stable Lévy process of index $\alpha \in(0,2]$ can be written as

$$
\begin{aligned}
& \psi_{\alpha}(\xi)=\int_{\mathbb{S}_{d}}|\langle\xi, y\rangle|^{\alpha}\left[1-i \operatorname{sgn}(\langle\xi, y\rangle) \tan \left(\frac{\pi \alpha}{2}\right)\right] \mathrm{M}(d y)+i\left\langle\xi, A_{0}\right\rangle \quad \text { if } \alpha \neq 1, \\
& \psi_{1}(\xi)=\int_{\mathbb{S}_{d}}|\langle\xi, y\rangle|\left[1+i \frac{\pi}{2} \operatorname{sgn}(\langle\xi, y\rangle) \log |\langle\xi, y\rangle|\right] \mathrm{M}(d y)+i\left\langle\xi, A_{0}\right\rangle,
\end{aligned}
$$

where the pair $\left(\mathrm{M}, A_{0}\right)$ is unique, and the measure $\mathrm{M}$, which depends on $v$ in (4.7), is called the spectral measure of $X$. See Samorodnitsky and Taqqu [48], pages 65-66.

Remark 4.2. Due to the space-homogeneity of Lévy processes, Theorem 3.3 and its proof can be simplified. First, we assume the following simpler conditions:

$\left(\mathrm{A} 2^{\prime \prime}\right)$ For any $\zeta>0$ and $T>0$, there exist positive constants $C_{1}, C_{2}, r_{0} \leq 1$ such that for all $0<t \leq T$ and $0<r \leq r_{0}$,

$$
\mathbb{P}(|X(t)| \leq r) \geq C_{1} \min \left\{1,\left(\frac{r}{t^{H-\zeta}}\right)^{d}\right\}
$$


and

$$
\mathbb{P}(|X(t)| \leq r) \leq C_{2} \min \left\{1,\left(\frac{r}{t^{H+\zeta}}\right)^{d}\right\} .
$$

$\left(\mathrm{A} 3^{\prime \prime}\right)$ We strengthen (A2") by assuming additionally that (4.9) holds for all $t>0$.

It is clear that the inequalities in (4.8) and (4.9) imply Condition (A2) with $J=\left\{\left(0, \zeta_{n}\right)\right\}$, where $\zeta_{n}$ is an arbitrary sequence with $\zeta_{n} \downarrow 0$ as $n \rightarrow \infty$. Second, instead of (3.15), we use directly Theorem 1.1 in Khoshnevisan [23] to get

$$
\mathbb{P}^{x}\left\{\inf _{t \leq s \leq T}|X(s)-x| \leq r\right\} \leq \frac{\int_{t}^{2 T} \mathbb{P}(|X(s)| \leq 2 r) d s}{\int_{0}^{T-t} \mathbb{P}(|X(s)| \leq r) d s}
$$

Note that, due to the space-homogeneity of $X$, the denominator in the right hand side of (4.10) is simpler that that in (3.15). One can check that a slightly modified version of (3.16) holds under condition (A2 $2^{\prime \prime}$ ). Hence, for a space-homogeneous Markov process that satisfies (A1), the conclusion of Theorem 3.3 still holds under either $1<H d$ and $\left(\mathrm{A} 2^{\prime \prime}\right)$; or $1=H d$ and $\left(\mathrm{A} 3^{\prime \prime}\right)$. We will use this modified version of Theorem 3.3 to Lévy processes.

As we mentioned earlier, for a Lévy process $X=\left\{X(t), t \in \mathbb{R}_{+}\right\}$, many of its sample path properties are characterized by the analytic or asymptotic properties of its characteristic exponent $\psi(\xi)$. In order to determine the Hausdorff and packing dimension of $X(E)$, we will make use of the following conditions:

(B3) There is a constant $\alpha \in(0,2]$ such that the following hold:

(i) If $0<\alpha<2$, then for every $\zeta^{\prime} \in(0,2-\alpha)$ we have

$$
K_{5}^{-1}|\xi|^{\alpha-\zeta^{\prime}} \leq \psi(\xi) \leq K_{5}|\xi|^{\alpha+\zeta^{\prime}}, \quad \forall \xi \in \mathbb{R}^{d} \text { with }|\xi| \geq \tau
$$

where $K_{5} \geq 1$ and $\tau$ are positive and finite constants.

(ii) If $\alpha=2$, then for any $\zeta^{\prime} \in(0,2)$,

$$
K_{5}^{-1}|\xi|^{2-\zeta^{\prime}} \leq \psi(\xi) \leq K_{5}|\xi|^{2}, \quad \forall \xi \in \mathbb{R}^{d} \text { with }|\xi| \geq \tau
$$

(B4) In addition to (B3), we assume that the left inequalities in (4.11) and (4.12) hold for all $\xi \in \mathbb{R}^{d}$.

Remark 4.3. The following are some remarks about Conditions (B3) and (B4).

(i) Since $\psi(\xi)$ is a negative definite function, the right inequality in (4.12) always holds for $|\xi| \geq 1$ (cf. Berg and Forst [5], page 46).

(ii) Conditions (4.11) and (4.12) are satisfied by a large class of symmetric Lévy processes whose Lévy measures have certain (approximate) regularly varying properties at the ori- 
gin. This can be explicitly formulated by modifying Condition (2.17) (use $|\lambda| \rightarrow 0$ instead of $|\lambda| \rightarrow \infty)$ and the proof of Theorem 2.5 in Xiao [57].

Now we are ready to state and prove the following theorem, which extends the uniform Hausdorff and packing dimension results of Hawkes [16], Hawkes and Pruitt [18], Perkins and Taylor [44] for stable Lévy processes to a class of symmetric Lévy processes.

Theorem 4.4. Let $X=\left\{X(t), t \in \mathbb{R}_{+}\right\}$be a symmetric Lévy process in $\mathbb{R}^{d}$ with exponent $\psi(\xi)$. We assume either (i) $1<\alpha d$ and (B3) hold; or (ii) $1=\alpha d$ and (B4) hold. Then with probability one,

$$
\operatorname{dim}_{\mathrm{H}} X(E)=\alpha \operatorname{dim}_{\mathrm{H}} E \quad \text { and } \quad \operatorname{dim}_{\mathrm{P}} X(E)=\alpha \operatorname{dim}_{\mathrm{P}} E
$$

for all Borel sets $E \subseteq \mathbb{R}_{+}$.

Proof. This theorem is a consequence of Theorem 3.3 and Remark 4.2. It is sufficient to verify that

(a) When $1<\alpha d$, (B3) implies Conditions (A1) and (A2") with $H=\frac{1}{\alpha}$.

(b) When $1=\alpha d$, (B4) implies Conditions (A1) and (A3") hold with $H=\frac{1}{\alpha}$.

It will be clear that (a) and (b) can be verified by the same method. For simplicity, we only show (a) in case (i) where $0<\alpha<2$ and leave the rest of the verification to an interested reader.

In order to verify Conditions (A1), we apply Proposition 3.2. For any fixed $\gamma \in(0,1 / \alpha)$, there exists $\epsilon_{0} \in(0,2-\alpha)$ such that $\gamma\left(\alpha+\epsilon_{0}\right)<1$. By (3.8), we know that for $t \in(0,1]$ small enough,

$$
\begin{aligned}
\sup _{x \in \mathbb{R}^{d}} \mathbb{P}^{x}\left\{\sup _{s \in[0, t]}|X(s)-x| \geq t^{\gamma}\right\} & \leq C t \sup _{|\xi| \leq t^{-\gamma}}|\psi(\xi)| \\
& \leq C t\left(\sup _{|\xi| \leq \tau}|\psi(\xi)|+K_{5} t^{-\gamma\left(\alpha+\epsilon_{0}\right)}\right) \\
& \leq C t^{1-\gamma\left(\alpha+\epsilon_{0}\right)} .
\end{aligned}
$$

Since $1-\gamma\left(\alpha+\epsilon_{0}\right)>0$, we see that Conditions (A1) holds with $\eta=1-\gamma\left(\alpha+\epsilon_{0}\right)$.

In order to verify Condition (A2 $2^{\prime \prime}$ ) under (4.11), we use an argument from Khoshnevisan and Xiao [25,27]. For any $r>0$, consider the nonnegative function

$$
\varphi_{r}(y)=\prod_{j=1}^{d} \frac{1-\cos \left(2 r y_{j}\right)}{2 \pi r y_{j}^{2}}, \quad \forall y \in \mathbb{R}^{d} .
$$

Its Fourier transform is given by

$$
\widehat{\varphi_{r}}(\xi)=\prod_{j=1}^{d}\left(1-\frac{\left|\xi_{j}\right|}{2 r}\right)^{+}, \quad \forall \xi \in \mathbb{R}^{d},
$$

where $a^{+}=\max (a, 0)$. 
Note that, for $\xi \in B(0, r)$, we have $1-(2 r)^{-1}\left|\xi_{j}\right| \geq \frac{1}{2}$. In light of (4.13), this implies $\mathbb{1}_{B(0, r)}(\xi) \leq 2^{d} \widehat{\varphi}_{r}(\xi)$, where $\mathbb{1}_{A}$ denotes the indicator function of the set $A$. On the other hand, if $\left|\xi_{j}\right| \geq 2 r$ for some $j \in\{1, \ldots, d\}$, by (4.13), then $\widehat{\varphi_{r}}(\xi)=0$. Hence, we have shown that for all $\forall \xi \in \mathbb{R}^{d}$,

$$
\mathbb{1}_{B(0, r)}(\xi) \leq 2^{d} \widehat{\varphi}_{r}(\xi) \leq 2^{d} \mathbb{1}_{B(0,2 \sqrt{d} r)}(\xi)
$$

Integrating the first inequality in (4.14) with respect to $v_{t}$, the distribution of $X(t)$, and using Parseval's formula yield

$$
\begin{aligned}
\mathbb{P}\{|X(t)| \leq r\} & \leq 2^{d} \int_{\mathbb{R}^{d}} \widehat{\varphi_{r}}(\xi) v_{t}(d \xi) \\
& =2^{d} \int_{\mathbb{R}^{d}} \varphi_{r}(\xi) \widehat{v_{t}}(\xi) d \xi \\
& =2^{d} \int_{\mathbb{R}^{d}} e^{-t \psi(\xi)} \prod_{j=1}^{d} \frac{1-\cos \left(2 r \xi_{j}\right)}{2 \pi r \xi_{j}^{2}} d \xi
\end{aligned}
$$

We split the last integral in (4.15) over $B(0, \tau)=\left\{\xi \in \mathbb{R}^{d}:|\xi|<\tau\right\}$ and its complement, respectively. For the first integral, we use the elementary inequality $1-\cos x \leq x^{2}(\forall x \in \mathbb{R})$, to derive

$$
\int_{|\xi| \leq \tau} e^{-t \psi(\xi)} \prod_{j=1}^{d} \frac{1-\cos \left(2 r \xi_{j}\right)}{2 \pi r \xi_{j}^{2}} d \xi \leq K r^{d} .
$$

For the second integral, we use (4.11), Parseval's formula and (4.14) to derive

$$
\begin{aligned}
\int_{|\xi|>\tau} e^{-t \psi(\xi)} \prod_{j=1}^{d} \frac{1-\cos \left(2 r \xi_{j}\right)}{2 \pi r \xi_{j}^{2}} d \xi & \leq \int_{\mathbb{R}^{d}} e^{-K_{5}^{-1} t\|\xi\|^{\alpha-\zeta^{\prime}}} \prod_{j=1}^{d} \frac{1-\cos \left(2 r \xi_{j}\right)}{2 \pi r \xi_{j}^{2}} d \xi \\
& =\int_{\mathbb{R}^{d}} \widehat{\varphi_{r}}(\xi) \mu_{t}(d \xi) \\
& \leq \int_{\mathbb{R}^{d}} \mathbb{1}_{B(0,2 \sqrt{d} r)}(\xi) \mu_{t}(d \xi) \\
& \leq C \min \left\{1,\left(\frac{r}{t^{1 /\left(\alpha-\zeta^{\prime}\right)}}\right)^{d}\right\} .
\end{aligned}
$$

In the above, $\mu_{t}$ denotes the distribution of the isotropic stable law with characteristic function $\widehat{\mu}_{t}(\xi)=e^{-K_{5}^{-1} t\|\xi\|^{\alpha-\zeta^{\prime}}}$ and the last inequality follows from the boundedness and scaling property of the density function of $\mu_{t}$. Combining (4.15)-(4.17) we derive that, for all $0<t \leq T$, (4.9) holds with $H=1 / \alpha$ and $\zeta=\frac{\zeta^{\prime}}{\alpha\left(\alpha-\zeta^{\prime}\right)}$. 
Next, we verify the lower bound in (4.8). Let $\tilde{r}=r /(2 \sqrt{d})$. It follows from (4.14) that

$$
\begin{aligned}
\mathbb{P}\{|X(t)| \leq r\} & \geq \int_{\mathbb{R}^{d}} \widehat{\varphi}_{\tilde{r}}(\xi) v_{t}(d \xi) \\
& =\int_{\mathbb{R}^{d}} \varphi_{\tilde{r}}(\xi) \widehat{v}_{t}(\xi) d \xi \\
& =\int_{\mathbb{R}^{d}} e^{-t \psi(\xi)} \prod_{j=1}^{d} \frac{1-\cos \left(2 \tilde{r} \xi_{j}\right)}{2 \pi \tilde{r} \xi_{j}^{2}} d \xi .
\end{aligned}
$$

Again, we split the last integral over $B(0, \tau)=\left\{\xi \in \mathbb{R}^{d}:|\xi|<\tau\right\}$ and its complement, and use a similar argument as in (4.17) to obtain

$$
\begin{aligned}
\mathbb{P}\{|X(t)| \leq r\} \geq & \int_{|\xi| \geq \tau} e^{-K_{5} t|\xi|^{\alpha+\xi^{\prime}}} \prod_{j=1}^{d} \frac{1-\cos \left(2 \tilde{r} \xi_{j}\right)}{2 \pi \tilde{r} \xi_{j}^{2}} d \xi \\
= & \int_{\mathbb{R}^{d}} e^{-K_{5} t|\xi|^{\alpha+\zeta^{\prime}}} \prod_{j=1}^{d} \frac{1-\cos \left(2 \tilde{r} \xi_{j}\right)}{2 \pi \tilde{r} \xi_{j}^{2}} d \xi \\
& -\int_{|\xi| \leq \tau} e^{-K_{5} t|\xi|^{\alpha+\zeta^{\prime}}} \prod_{j=1}^{d} \frac{1-\cos \left(2 \tilde{r} \xi_{j}\right)}{2 \pi \tilde{r} \xi_{j}^{2}} d \xi \\
\geq & C \min \left\{1,\left(\frac{r}{t^{1 /\left(\alpha+\zeta^{\prime}\right)}}\right)^{d}\right\}
\end{aligned}
$$

Note that $\frac{1}{\alpha+\zeta^{\prime}}=\frac{1}{\alpha}-\frac{\zeta^{\prime}}{\alpha\left(\alpha+\zeta^{\prime}\right)}>\frac{1}{\alpha}-\zeta$. It follows from (4.19) that we can choose a constant $C_{1}$ such that for all $0<t \leq T$, the lower bound in (4.8) holds with $H=1 / \alpha$ and $\zeta=\frac{\zeta^{\prime}}{\alpha\left(\alpha-\zeta^{\prime}\right)}$. Hence, we have shown that (4.11) implies Condition (A2") with $H=1 / \alpha$ and $\zeta=\frac{\zeta^{\prime}}{\alpha\left(\alpha-\zeta^{\prime}\right)}$. This completes the proof of Theorem 4.4 .

Remark 4.5. Our method for verifying of Condition (A2") provides a comparison theorem for the transition probabilities of Lévy processes in terms of their Lévy exponents. This may be of independent interest. Several authors have established estimates on the transition density functions of Lévy processes based on information on their Lévy measures or Lévy exponents; see Kaleta and Sztonyk [21] and the references therein for further information.

\subsection{Stable jump-diffusions}

Let $X=\left\{X(t), t \in \mathbb{R}_{+}, \mathbb{P}^{x}, x \in \mathbb{R}^{d}\right\}$ be a Feller process with values in $\mathbb{R}^{d}$ corresponding to the Feller semigroup defined by the following equation:

$$
\frac{\partial u}{\partial t}=\left\langle A(x), \frac{\partial u}{\partial x}\right\rangle+\int_{0}^{\infty} \int_{\mathbb{S}_{d}}\left(u(x+|\xi| s)-u(x)-\frac{\left\langle|\xi| s, \frac{\partial u}{\partial x}(x)\right\rangle}{1+|\xi|^{2}}\right) \frac{d|\xi|}{|\xi|^{1+\alpha}} \tilde{\mathrm{M}}(x, d s),
$$


where the drift $A$ and the spectral measure $\tilde{M}$ on $\mathbb{S}_{d}$ depend smoothly on $x$, see Theorem 3.1 of Kolokoltsov [35] for the precise conditions on $A$ and $\widetilde{M}$.

Following Kolokoltsov [35], we call $X$ a stable jump-diffusion. Roughly speaking, these are the processes corresponding to stable Lévy processes in the same way as the ordinary diffusions corresponding to Brownian motion.

Locally, the stable jump-diffusion $X$ resembles a stable Lévy process, hence it is expected that a stable jump-diffusion has sample path properties similar to those of a stable Lévy process. Some of these properties such as the limsup behavior of $X(t)$ as $t \rightarrow 0$ have been established by Kolokoltsov [35], Section 6. Moreover, for every fixed Borel set $E \subseteq \mathbb{R}_{+}$, the Hausdorff dimension of the image set $X(E)$ can be derived from Theorem 4.14 in Xiao [56].

The following theorem proves a uniform Hausdorff and packing dimension result for stable jump diffusions.

Theorem 4.6. Let $X=\left\{X(t), t \in \mathbb{R}_{+}, \mathbb{P}^{x}\right\}$ be a stable jump-diffusion in $\mathbb{R}^{d}$ with index $\alpha \in(0,2]$ as described above. If $\alpha \leq d$, then for every $x \in \mathbb{R}^{d}, \mathbb{P}^{x}$-almost surely

$$
\operatorname{dim}_{\mathrm{H}} X(E)=\alpha \operatorname{dim}_{\mathrm{H}} E \quad \text { and } \quad \operatorname{dim}_{\mathrm{P}} X(E)=\alpha \operatorname{dim}_{\mathrm{P}} E
$$

hold for all Borel sets $E \subseteq \mathbb{R}_{+}$.

Proof. It follows from Theorem 6.1 of Kolokoltsov [35] that Condition (A1) holds for $X$ with $H=1 / \alpha$. On the other hand, Theorem 3.1 of Kolokoltsov [35] implies that (A2) with $H=1 / \alpha$, $\varepsilon=0$ and $\zeta=0$. Hence, (4.21) follows immediately from Theorem 3.3.

Remark 4.7. We remark that one can also apply the inequality (3.8) to verify Condition (A1) for this case. By (1.9) of Kolokoltsov [35], the symbol of the stable jump diffusion defined by (4.20) has the form:

$$
q(x, \xi)=i(A(x), \xi)-\int_{\mathbb{S}_{d}}|(\xi, s)|^{\alpha} M(x, d s) .
$$

Moreover, we assume that same conditions on $A$ and $M$ as in Theorem 3.1 in Kolokoltsov [35], which contain

- there exist positive constants $C_{1}$ and $C_{2}$ such that

$$
C_{1}|\xi|^{\alpha} \leq \int_{\mathbb{S}_{d}}|(\xi, s)|^{\alpha} M(x, d s) \leq C_{2}|\xi|^{\alpha},
$$

- $A$ is uniformly bounded in $x$, that is, $\sup _{x \in \mathbb{R}^{d}}|A(x)|<\infty$,

- $A(x) \equiv 0$ for $\alpha \leq 1$.

Then, by (3.8), for any $\gamma \in(0,1 / \alpha)$, we have, for all $x \in \mathbb{R}^{d}$ and $t \in[0,1]$,

$$
\mathbb{P}^{x}\left\{\sup _{s \in[0, t]}\left|X_{s}-x\right| \geq t^{\gamma}\right\} \leq C t \sup _{|y-x| \leq t^{\gamma}} \sup _{|\xi| \leq t^{-\gamma}}|q(y, \xi)|
$$




$$
\begin{aligned}
& \leq C t \sup _{|\xi| \leq t^{-\gamma}}\left(\sup _{x \in \mathbb{R}^{d}}|A(x)||\xi|+C_{2}|\xi|^{\alpha}\right) \\
& \leq C t^{1-\gamma \alpha}\left(\sup _{x \in \mathbb{R}^{d}}|A(x)|+C_{2}\right) .
\end{aligned}
$$

This verifies Condition (A1) with $\eta=1-\gamma \alpha$.

\subsection{Non-symmetric stable-type processes}

Let $X=\left\{X(t), t \in \mathbb{R}_{+}\right\}$be a pure jump process such that its infinitesimal generator has the following form:

$$
\mathcal{L}_{\alpha}^{\kappa} f(x):=\lim _{\varepsilon \rightarrow 0} \int_{\left\{z \in \mathbb{R}^{d}:|z| \geq \varepsilon\right\}}(f(x+z)-f(x)) \frac{\kappa(x, z)}{|z|^{d+\alpha}} d z,
$$

where $d \geq 1,0<\alpha<2$, and $\kappa(x, z)$ is a measurable function on $\mathbb{R}^{d} \times \mathbb{R}^{d}$ satisfying

$$
0<\kappa_{0} \leq \kappa(x, z) \leq \kappa_{1}, \quad \kappa(x, z)=\kappa(x,-z),
$$

and for some $\beta \in(0,1)$

$$
|\kappa(x, z)-\kappa(y, z)| \leq \kappa_{2}|x-y|^{\beta} .
$$

This class of Markov processes has been studied by Chen and Kumagai [11], Chen and Zhang [12], among others.

The following uniform dimension result is a consequence of Theorem 3.3.

Theorem 4.8. Let $X=\left\{X(t), t \in \mathbb{R}_{+}\right\}$be an non-symmetric $\alpha$-stable-type Markov process defined above. If $1 \leq \alpha d$, then, for every $x \in \mathbb{R}^{d}, \mathbb{P}^{x}$-almost surely

$$
\operatorname{dim}_{\mathrm{H}} X(E)=\alpha \operatorname{dim}_{\mathrm{H}} E \quad \text { and } \operatorname{dim}_{\mathrm{P}} X(E)=\alpha \operatorname{dim}_{\mathrm{P}} E
$$

hold for all Borel sets $E \subseteq \mathbb{R}_{+}$.

Proof. By Chen and Zhang [12], $X_{t}$ has a Hölder continuous transition density function $p(t, x, y)$. Furthermore, there are positive constants $C_{5}$ and $C_{6}$ depending on $d, \alpha, \beta, \kappa_{0}, \kappa_{1}$, $\kappa_{2}$ such that for all $t \in(0,1], x, y \in \mathbb{R}^{d}$,

$$
C_{5} t\left(t^{1 / \alpha}+|x-y|\right)^{-d-\alpha} \leq p(t, x, y) \leq C_{6} t\left(t^{1 / \alpha}+|x-y|\right)^{-d-\alpha},
$$

which is equivalent to

$$
C_{7} \min \left\{t^{-d / \alpha}, \frac{t}{|x-y|^{d+\alpha}}\right\} \leq p(t, x, y) \leq C_{8} \min \left\{t^{-d / \alpha}, \frac{t}{|x-y|^{d+\alpha}}\right\},
$$

where $C_{7}$ and $C_{8}$ are two positive constants depending on $d, \alpha, \beta, \kappa_{0}, \kappa_{1}, \kappa_{2}$. 
Define

$$
P_{1}(t, x, A):=C_{7} \int_{A} \min \left\{t^{-d / \alpha}, \frac{t}{|x-y|^{d+\alpha}}\right\} d y
$$

and

$$
P_{2}(t, x, A):=C_{8} \int_{A} \min \left\{t^{-d / \alpha}, \frac{t}{|x-y|^{d+\alpha}}\right\} d y .
$$

Both $P_{1}$ and $P_{2}$ have $1 / \alpha$-self-similar property, that is, for any $r>0$

$$
P_{i}(t, x, A)=P_{i}\left(r t, r^{1 / \alpha} x, r^{1 / \alpha} A\right), \quad i=1,2 .
$$

Indeed, for all $A \in \mathcal{B}\left(\mathbb{R}^{d}\right)$ and $r>0$, we have

$$
\begin{aligned}
& \int_{r^{1 / \alpha} A} \min \left\{(r t)^{-d / \alpha}, \frac{r t}{\left|r^{1 / \alpha} x-y\right|^{d+\alpha}}\right\} \mathrm{d} y \\
& =\int_{A} \min \left\{(r t)^{-d / \alpha}, \frac{r t}{r^{(d+\alpha) / \alpha}|x-z|^{d+\alpha}}\right\} r^{d / \alpha} \mathrm{d} z \\
& =\int_{A} \min \left\{t^{-d / \alpha}, \frac{t}{|x-z|^{d+\alpha}}\right\} \mathrm{d} z .
\end{aligned}
$$

By a straightforward computation, there exist positive constants $C_{9}$ and $C_{10}$ such that

$$
P_{1}(1, y, B(x, r)) \geq C_{9} \min \left\{1, r^{d}\right\}, \quad \forall|y-x| \leq r, r>0,
$$

and

$$
P_{2}(1, x, B(x, r)) \leq C_{10} \min \left\{1, r^{d}\right\}, \quad \forall x \in \mathbb{R}^{d}, r>0 .
$$

Then for all $|y-x| \leq r$,

$$
\begin{aligned}
P(t, y, B(x, r)) & \geq P_{1}(t, y, B(x, r)) \\
& =P_{1}\left(1, t^{-\frac{1}{\alpha}} y, B\left(t^{-\frac{1}{\alpha}} x, t^{-\frac{1}{\alpha}} r\right)\right) \\
& \geq C_{9} \min \left\{1,\left(\frac{r}{t^{\frac{1}{\alpha}}}\right)^{d}\right\}
\end{aligned}
$$

and $\forall r>0$,

$$
\begin{aligned}
P(t, x, B(x, r)) & \leq P_{2}(t, x, B(x, r)) \\
& =P_{2}\left(1, t^{-\frac{1}{\alpha}} x, B\left(t^{-\frac{1}{\alpha}} x, t^{-\frac{1}{\alpha}} r\right)\right) \\
& \leq C_{10} \min \left\{1,\left(\frac{r}{t^{\frac{1}{\alpha}}}\right)^{d}\right\} .
\end{aligned}
$$

Thus, Condition (A2) holds. 
Next, we verify the condition (A1). As in Remark 4.7, this can be done by applying Proposition 3.2 and the fact that the symbol $q$ of $X$ has the form

$$
q(x, \xi)=\int_{\mathbb{R}^{d} \backslash\{0\}}\left(1-e^{i z \cdot \xi}+i z \cdot \xi 1_{(0,1]}(|z|)\right) \frac{\kappa(x, z)}{|z|^{d+\alpha}} d z,
$$

which can be bounded from above by $C|\xi|^{\alpha}$. Here we provide a different proof.

By the Lévy-Itô decomposition, one has

$$
X_{t}=X_{0}+\int_{0}^{t} \int_{|y| \leq l} y \tilde{N}\left(X_{s-}, d y, d s\right)+\int_{0}^{t} \int_{|y|>l} y N\left(X_{s-}, d y, d s\right),
$$

where $l$ can be any positive constant, $N(x, d y, d s)$ is the Poisson random measure with the intensity measure $v(x, d y) d s:=\frac{\kappa(x, y)}{|y|^{d+\alpha}} d y d s$ and $\tilde{N}\left(X_{s-}, d y, d s\right)=N\left(X_{s-}, d y, d s\right)-$ $v\left(X_{s-}, d y\right) d s$ is the compensated Poisson random measure.

Since $\alpha \in(0,2)$, we can find some $p$ satisfying $0<p \leq 1$ and $p<\alpha<2 p$. For some $l>0$ and $x \in \mathbb{R}^{d}$, we define a smooth function $f$ on $\mathbb{R}^{d}$ by

$$
f(y)=\left(|y-x|^{2}+l^{2}\right)^{p / 2}, \quad y \in \mathbb{R}^{d},
$$

it is easy to check that for all $x, y \in \mathbb{R}^{d}$,

$$
\left|f\left(y_{1}\right)-f\left(y_{2}\right)\right| \leq\left|y_{1}-y_{2}\right|^{p} .
$$

Now let $X_{0}=x$, by Itô's formula (Applebaum [2], Section 4.4.2), we get, for any $l>0$,

$$
\begin{aligned}
f\left(X_{t}\right)= & f(x)+\int_{0}^{t} \int_{|y| \leq l}\left[f\left(X_{s-}+y\right)-f\left(X_{s-}\right)\right] \tilde{N}\left(X_{s-}, d y, d s\right) \\
& +\int_{0}^{t} \int_{|y| \leq l}\left[f\left(X_{s-}+y\right)-f\left(X_{s-}\right)-\left\langle\nabla f\left(X_{s-}\right), y\right\rangle\right] v\left(X_{s-}, d y\right) d s \\
& +\int_{0}^{t} \int_{|y|>l}\left[f\left(X_{s-}+y\right)-f\left(X_{s-}\right)\right] N\left(X_{s-}, d y, d s\right) \\
= & : l^{p}+I_{1}(t)+I_{2}(t)+I_{3}(t) .
\end{aligned}
$$

Take $l=T^{1 / \alpha}$, for any $T>0$, by Burkholder's inequality and (4.24), we have

$$
\begin{aligned}
\mathbb{E}\left(\sup _{0 \leq t \leq T}\left|I_{1}(t)\right|\right) & \leq \mathbb{E}\left[\int_{0}^{T} \int_{|y| \leq T^{1 / \alpha}}|y|^{2 p} N\left(X_{s-}, d y, d s\right)\right]^{1 / 2} \\
& \leq\left[\int_{0}^{T} \int_{|y| \leq T^{1 / \alpha}}|y|^{2 p} \frac{\kappa_{1}}{|y|^{d+\alpha}} d y d s\right]^{1 / 2} \\
& \leq C T^{p / \alpha} .
\end{aligned}
$$


For $I_{2}(t)$, by Taylor's expansion, we have

$$
f\left(X_{s-}+y\right)-f\left(X_{s-}\right)-\left\langle\nabla f\left(X_{s-}\right), y\right\rangle=\left\langle y, \nabla^{2} f\left(X_{s-}+\theta y\right) y\right\rangle,
$$

where $\theta \in[0,1]$ depending on $X_{s-}$ and $y$, one can verify that

$$
\begin{aligned}
\mid\langle y & \left., \nabla^{2} f\left(X_{s-}+\theta y\right) y\right\rangle \mid \\
& =\left|\frac{p|y|^{2}}{\left(T^{2 / \alpha}+\left|X_{s-}+\theta y-x\right|^{2}\right)^{1-\frac{p}{2}}}+\frac{p(p-2)\left|\left\langle y, X_{s-}+\theta y-x\right\rangle\right|^{2}}{\left(T^{2 / \alpha}+\left|X_{s-}+\theta y-x\right|^{2}\right)^{2-\frac{p}{2}}}\right| \\
& \leq \frac{p|y|^{2}}{T^{\frac{2}{\alpha}\left(1-\frac{p}{2}\right)}+\frac{p(2-p)|y|^{2}\left|X_{s-}+\theta y-x\right|^{2}}{\left(T^{2 / \alpha}+\left|X_{s-}+\theta y-x\right|^{2}\right)^{2-\frac{p}{2}}}} \\
& \leq \frac{\left(3 p-p^{2}\right)|y|^{2}}{T^{\frac{2}{\alpha}\left(1-\frac{p}{2}\right)}} .
\end{aligned}
$$

Hence,

$$
\begin{aligned}
\mathbb{E}\left(\sup _{0 \leq t \leq T}\left|I_{2}(t)\right|\right) & \leq C T^{\frac{2}{\alpha}\left(\frac{p}{2}-1\right)} \int_{0}^{T} \mathbb{E} \int_{|y| \leq T^{1 / \alpha}}|y|^{2} v\left(X_{s-}, d y\right) d s \\
& \leq C T^{\frac{2}{\alpha}\left(\frac{p}{2}-1\right)} \int_{0}^{T} \int_{|y| \leq T^{1 / \alpha}} \frac{\kappa_{1}|y|^{2}}{|y|^{d+\alpha}} d y d s \\
& \leq C T^{p / \alpha} .
\end{aligned}
$$

For $I_{3}(t)$, by (4.24) again, we get

$$
\begin{aligned}
\mathbb{E}\left(\sup _{0 \leq t \leq T}\left|I_{3}(t)\right|\right) & \leq \mathbb{E} \int_{0}^{T} \int_{|y|>T^{1 / \alpha}}|y|^{p} v\left(X_{s-}, d y\right) d s \\
& \leq \int_{0}^{T} \int_{|y|>T^{1 / \alpha}} \frac{\kappa_{1}|y|^{p}}{|y|^{d+\alpha}} d y d s \\
& \leq C T^{p / \alpha}
\end{aligned}
$$

Combining (4.25)-(4.27) yields

$$
\mathbb{E}^{x}\left(\sup _{t \in[0, T]}\left|\left(\left|X_{t}-x\right|^{2}+T^{\frac{2}{\alpha}}\right)^{p / 2}-T^{\frac{p}{\alpha}}\right|\right) \leq C T^{p / \alpha} .
$$

Hence, for any $r>\alpha$,

$$
\mathbb{P}^{x}\left\{\sup _{0 \leq s \leq t}\left|X_{s}-x\right| \geq t^{1 / r}\right\} \leq \frac{\mathbb{E}^{x}\left[\sup _{s \in[0, t]}\left|X_{s}-x\right|^{p}\right]}{t^{p / r}}
$$




$$
\begin{aligned}
& \leq \frac{\mathbb{E}^{x}\left[\sup _{s \in[0, t]} \mid\left(\left|X_{s}-x\right|^{2}+t^{\frac{2}{\alpha}}\right)^{p / 2}-t^{\frac{p}{\alpha}}\right]+t^{\frac{p}{\alpha}}}{t^{p / r}} \\
& \leq C t^{p / \alpha-p / r} .
\end{aligned}
$$

Thus Condition (A1) also holds. Hence, the conclusion of Theorem 4.8 follows from Theorem 3.3.

\section{Acknowledgements}

We thank the referee for his or her valuable comments and, in particular, for suggesting the use of Proposition 3.2, which has helped to simplify the proof of Theorem 4.4 and improve the quality of this paper. This work was conducted during the first author visited the Department of Mathematics, Faculty of Science and Technology, University of Macau, he thanks for the finance support and hospitality. Xiaobin Sun is supported by the National Natural Science Foundation of China (11601196), Natural Science Foundation of the Higher Education Institutions of Jiangsu Province (16KJB110006) and the Project Funded by the Priority Academic Program Development of Jiangsu Higher Education Institutions. Y. Xiao is partially supported by NSF grants DMS-1612885 and DMS-1607089. Lihu Xu is supported by the following grants: NNSFC 11571390, Macau S.A.R. FDCT 030/2016/A1 and FDCT 038/2017/A1, University of Macau MYRG (2015-00021-FST, 2016-00025-FST). Jianliang Zhai is supported by the National Natural Science Foundation of China (11431014, 11401557).

\section{References}

[1] Alili, L., Chaumont, L., Graczyk, P. and Żak, T. (2017). Inversion, duality and Doob $h$-transforms for self-similar Markov processes. Electron. J. Probab. 22 Paper No. 20. DOI:10.1214/17-EJP33. MR3622890

[2] Applebaum, D. (2004). Lévy Processes and Stochastic Calculus. Cambridge Studies in Advanced Mathematics 93. Cambridge: Cambridge Univ. Press. MR2072890

[3] Bass, R. (1988). Uniqueness in law for pure jump Markov processes. Probab. Theory Related Fields 79 271-287.

[4] Benjamini, I., Chen, Z.-Q. and Rohde, S. (2004). Boundary trace of reflecting Brownian motions. Probab. Theory Related Fields 129 1-17. DOI:10.1007/s00440-003-0318-7. MR2052860

[5] Berg, C. and Forst, G. (1975). Potential Theory on Locally Compact Abelian Groups. Ergebnisse der Mathematik und ihrer Grenzgebiete 87. New York: Springer. MR0481057

[6] Bertoin, J. and Yor, M. (2005). Exponential functionals of Lévy processes. Probab. Surv. 2 191-212. DOI:10.1214/154957805100000122. MR2178044

[7] Blumenthal, R.M. and Getoor, R. (1960). A dimension theorem for sample functions of stable processes. Illinois J. Math. 4 370-375.

[8] Böttcher, B., Schilling, R. and Wang, J. (2013). Lévy Matters. III. Lévy-Type Processes: Construction, Approximation and Sample Path Properties. Lecture Notes in Math. 2099. Cham: Springer.

[9] Chaumont, L., Pantí, H. and Rivero, V. (2013). The Lamperti representation of real-valued self-similar Markov processes. Bernoulli 19 2494-2523. 
[10] Chen, Z.Q. (2009). Symmetric jump processes and their heat kernel estimates. Sci. China Math. 52 $1423-1445$.

[11] Chen, Z.Q. and Kumagai, T. (2003). Heat kernel estimates for stable-like processes on $d$-sets. Stoch. Model. Appl. 108 27-62.

[12] Chen, Z.Q. and Zhang, X. (2016). Heat kernels and analyticity of non-symmetric jump diffusion semigroups. Probab. Theory Related Fields $165267-312$.

[13] Falconer, K.J. (2003). Fractal Geometry-Mathematical Foundations and Applications, 2nd ed. New York: Wiley \& Sons.

[14] Gikhman, I.I. and Skorohod, A.V. (1974). The Theory of Stochastic Processes, Vol. 1. Berlin: Springer.

[15] Graversen, S.E. and Vuolle-Apiala, J. (1986). $\alpha$-Self-similar Markov processes. Probab. Theory Related Fields 71 149-158.

[16] Hawkes, J. (1970/71). Some dimension theorems for the sample functions of stable processes. Indiana Univ. Math. J. 20 733-738. DOI:10.1512/iumj.1971.20.20058. MR0292164

[17] Hawkes, J. (1971). On the Hausdorff dimension of the intersection of the range of a stable process with a Borel set. Z. Wahrsch. Verw. Gebiete 19 90-102.

[18] Hawkes, J. and Pruitt, W.E. (1973/74). Uniform dimension results for processes with independent increments. Z. Wahrsch. Verw. Gebiete 28 277-288.

[19] Jacob, N. (2001). Pseudo Differential Operators and Markov Processes, Vol. I: Fourier Analysis and Semigroups. London: Imperial College Press. MR1873235

[20] Jacob, N. and Schilling, R.L. (2001). Lévy-type processes and pseudodifferential operators. In Lévy Processes 139-168. Boston, MA: Birkhäuser. MR1833696

[21] Kaleta, K. and Sztonyk, P. (2015). Estimates of transition densities and their derivatives for jump Lévy processes. J. Math. Anal. Appl. 431 260-282.

[22] Kaufman, R. (1968). Une propriété métrique du mouvement brownien. C. R. Acad. Sci. Paris 268 $727-728$.

[23] Khoshnevisan, D. (1997). Escape rates for Lévy processes. Studia Sci. Math. Hungar. 33 177-183.

[24] Khoshnevisan, D., Schilling, R.L. and Xiao, Y. (2012). Packing dimension profiles and Lévy processes. Bull. Lond. Math. Soc. 44 931-943.

[25] Khoshnevisan, D. and Xiao, Y. (2003). Weak unimodality of finite measures, and an application to potential theory of additive Lévy processes. Proc. Amer. Math. Soc. 131 2611-2616. DOI:10.1090/ S0002-9939-02-06778-3. MR1974662

[26] Khoshnevisan, D. and Xiao, Y. (2005). Lévy processes: Capacity and Hausdorff dimension. Ann. Probab. 33 841-878.

[27] Khoshnevisan, D. and Xiao, Y. (2008). Packing dimension of the range of a Lévy process. Proc. Amer. Math. Soc. 136 2597-2607. DOI:10.1090/S0002-9939-08-09163-6. MR2390532

[28] Khoshnevisan, D. and Xiao, Y. (2009). Harmonic analysis of additive Lévy processes. Probab. Theory Related Fields 145 459-515.

[29] Khoshnevisan, D. and Xiao, Y. (2015). Brownian motion and thermal capacity. Ann. Probab. 43 405434.

[30] Kinney, J.R. (1953). Continuity properties of sample functions of Markov processes. Trans. Amer. Math. Soc. 74 280-302. DOI:10.2307/1990883. MR0053428

[31] Kiu, S.W. (1980). Semi-stable Markov processes in $\mathbf{R}^{n}$. Stoch. Model. Appl. 10 183-191.

[32] Knopova, V. and Kulik, A. (2017). Intrinsic compound kernel estimates for the transition probability density of a Lévy-type process and their applications. Probab. Math. Statist. 37 53-100.

[33] Knopova, V. and Schilling, R. (2015). On level and collision sets of some Feller processes. ALEA Lat. Am. J. Probab. Math. Stat. 12 1001-1029.

[34] Knopova, V., Schilling, R.L. and Wang, J. (2015). Lower bounds of the Hausdorff dimension for the images of Feller processes. Statist. Probab. Lett. 97 222-228. DOI:10.1016/j.spl.2014.11.027. MR3299774 
[35] Kolokoltsov, V. (2000). Symmetric stable laws and stable-like jump-diffusions. Proc. Lond. Math. Soc. $80725-768$.

[36] Kolokoltsov, V. (2010). Nonlinear Markov Processes and Kinetic Equations. Cambridge Tracts in Mathematics 182. Cambridge: Cambridge Univ. Press.

[37] Kühn, F. (2017). Existence and estimates of moments for Lévy-type processes. Stochastic Process. Appl. 127 1018-1041.

[38] Kühn, F. (2017). Lévy Matters IV. Lévy-Type Processes: Moments, Construction and Heat Kernel Estimates. Lecture Notes in Math. 2187. Berlin: Springer.

[39] Lamperti, J. (1972). Semi-stable Markov processes. Z. Wahrsch. Verw. Gebiete 22 205-225.

[40] Liu, L. and Xiao, Y. (1998). Hausdorff dimension theorems for self-similar Markov processes. Probab. Math. Statist. 18 369-383. MR1671608

[41] Manstavičius, M. (2004). p-Variation of strong Markov processes. Ann. Probab. 32 2053-2066.

[42] Mörters, P. and Peres, P. (2010). Brownian Motion. Cambridge: Cambridge Univ. Press.

[43] Negoro, A. (1994). Stable-like processes: Construction of the transition density and the behavior of sample paths near $t=0$. Osaka J. Math. 31 189-214.

[44] Perkins, E.A. and Taylor, S.J. (1987). Uniform measure results for the image of subsets under Brownian motion. Probab. Theory Related Fields 76 257-289. DOI:10.1007/BF01297485. MR0912654

[45] Pruitt, W.E. (1975). Some dimension results for processes with independent increments. In Stochastic Processes and Related Topics (Proc. Summer Res. Inst. on Statist. Inference for Stochastic Processes) 133-165. New York: Academic Press.

[46] Pruitt, W.E. (1981). The growth of random walks and Lévy processes. Ann. Probab. 9 948-956.

[47] Revuz, D. and Yor, M. (1994). Continuous Martingales and Brownian Motion, 2nd ed. Berlin: Springer.

[48] Samorodnitsky, G. and Taqqu, M.S. (1994). Stable Non-Gaussian Random Processes: Stochastic Models with Infinite Variance. Stochastic Modeling. New York: Chapman \& Hall. MR1280932

[49] Schilling, R.L. (1998). Growth and Hölder conditions for the sample paths of Feller processes. Probab. Theory Related Fields 112 565-611.

[50] Schilling, R.L. and Partzsch, L. (2014). Brownian Motion. An Introduction to Stochastic Processes, 2nd ed. Berlin: De Gruyter. xvi+408 pp.

[51] Schilling, R.L. and Schnurr, A. (2010). The symbol associated with the solution of a stochastic differential equation. Electron. J. Probab. 15 1369-1393.

[52] Taylor, S.J. (1986). The measure theory of random fractals. Math. Proc. Cambridge Philos. Soc. 100 383-406. DOI:10.1017/S0305004100066160. MR0857718

[53] Vuolle-Apiala, J. (1994). Itô excursion theory for self-similar Markov processes. Ann. Probab. 22 $546-565$.

[54] Vuolle-Apiala, J. and Graversen, S.E. (1988). Duality theory for self-similar Markov processes. Ann. Inst. Henri Poincaré Probab. Stat. 22 376-392.

[55] Xiao, Y. (1998). Asymptotic results for self-similar Markov processes. In Asymptotic Methods in Probability and Statistics 323-340. Amsterdam: North-Holland.

[56] Xiao, Y. (2004). Random fractals and Markov processes. In Fractal Geometry and Applications: A Jubilee of Benoît Mandelbrot, Part 2. Proc. Sympos. Pure Math., Part 272 261-338. Providence, RI: Amer. Math. Soc. MR2112126

[57] Xiao, Y. (2008). Strong local nondeterminism and sample path properties of Gaussian random fields. In Asymptotic Theory in Probability and Statistics with Applications (T.L. Lai, Q. Shao, L. Qian, eds.). Adv. Lect. Math. (ALM) 2 136-176. Somerville, MA: Int. Press. MR2466984

[58] Yang, X. (2005). Sharp value for the Hausdorff dimension of the range and the graph of stable-like processes. Bernoulli. To appear.

Received July 2017 and revised September 2017 